\title{
Elastic damage to crack transition in a coupled non-local implicit discontinuous Galerkin/extrinsic cohesive law framework
}

\author{
L. $\mathrm{Wu}^{\mathrm{a}}$, G. Becker ${ }^{\mathrm{b}}$, L. Noels ${ }^{\mathrm{a}, *}$ \\ ${ }^{a}$ University of Liège - Department of Aerospace and Mechanical Engineering \\ Computational \& Multiscale Mechanics of Materials, Chemin des Chevreuils 1, B-4000 Liège, Belgium \\ ${ }^{b}$ Massachusetts Institute of Technology, Department of Aeronautics and Astronautics \\ 77 Massachusetts Avenue, Cambridge, MA 02139-4307, United States
}

\begin{abstract}
One current challenge related to computational fracture mechanics is the modeling of ductile fracture and in particular the damage to crack transition. On the one hand, continuum damage models, especially in their non-local formulation which avoids the loss of solution uniqueness, can capture the material degradation process up to the localization of the damage, but are unable to represent a discontinuity in the structure. On the other hand cohesive zone methods can represent the process zone at the crack tip governing the crack propagation, but cannot account for the diffuse material damaging process.

In this paper we propose to combine, in a small deformations setting, a non-local elastic damage model with a cohesive zone model. This combination is formulated within a discontinuous Galerkin finite element discretization. Indeed this DG weak formulation can easily be developed in a non-local implicit form and naturally embeds interface elements that can be used to integrate the traction separation law of the cohesive zone model. The method remains thus consistent and computationally efficient as compared to other cohesive element approaches.

The effects of the damage to crack transition and of the mesh discretization are respectively studied on the compact tension specimen and on the double-notched specimen, demonstrating the efficiency and accuracy of the method.
\end{abstract}

Keywords: Continuous damage mechanics, fracture, cohesive zone method, damage to crack transition, discontinuous Galerkin method

\section{Introduction}

Two approach families dedicated to failure analyzes of ductile materials are traditionally used: the continuous and discontinuous approaches. Continuous approaches, as the continuous damage mechanics $(\mathrm{CDM})[1,2,3, e . g]$, describe the material degradation process through the evolution of internal variables. An important issue of CDM approaches in their local form is that strain-softening leads to an ill-posed problem, and consequently the numerical results suffer from pathological localization and strong meshdependency. This ill-posedness can be avoided by applying some regularization techniques, as a non-local model $[4,5,6,7,8$, e.g. $]$ or a gradient enhanced model [9]. On the contrary, discontinuous approaches, which are typically used in fracture mechanics, model the crack in a discrete way. In uncracked parts of the body, the mechanical properties of the material are assumed intact, i.e. the degradation and softening of the material are not considered, while this degradation is modeled through the crack propagation. The progressive degradation process occurring in the process zone at crack tip can be modeled by the cohesive zone model (CZM) $[10,11]$ through the shape of the cohesive law, also called traction separation law (TSL),

\footnotetext{
*Corresponding author, Phone: +324366 48 26, Fax: +3243669505

Email address: L.Noels@ulg.ac.be (L. Noels)
} 
which describes the irreversible evolution of the traction exerted between the crack lips in terms of the crack opening.

Both continuous and discontinuous models can be considered in the finite element (FE) framework. On the one hand, with continuous approaches, a classical FE discretization (possibly formulated in a nonlocal way) can be directly used. On the other hand, when dealing with discontinuous approaches as the CZM, the FE discretization requires to be modified to account for a description of the crack. The three most popular methods to represent the crack are (i) the extended finite element method (xFEM) [12, 13], (ii) the embedded localization method (EFEM) [14], and (iii) the use of interface elements. For the two first approaches, the crack can be represented in an arbitrary existing FE mesh through global or local enrichment and can also integrate a CZM [15]. For the third approach, the crack propagates at the boundary of adjacent FEs and is modeled by the insertion of interface or cohesive elements [16]. When an assumed criterion is reached, the cohesive elements allow shifting from a continuous form into a discontinuous one to integrate the TSL. The TSL can be intrinsic in which case the cohesive elements are inserted since the very beginning of the simulation, and they should thus represents the pre-cracked stage under the form of a penalty response $[17,18]$, which makes the method not consistent and suffering from a mesh dependency [19]. This has motivated the use of extrinsic cohesive laws (ECL) which represent the fracturing response only, and for which cohesive elements are inserted at the fracture onset [20, 21]. Nevertheless the computational efficiency of the extrinsic approach is still challenging for 3D parallel applications as it requires mesh topology modifications on the fly. An energetically rigorous and computationally efficient way to introduce a CZM is to combine the extrinsic cohesive law with a discontinuous Galerkin (DG) approach [22, 23, 24, 25, 26, 27]. With this hybrid DG/ECL method, interface elements are inserted between bulk elements at the beginning of the simulation, but the consistency and continuity during the pre-fracture stage are ensured by having recourse to the DG interface terms, contrarily to a classical intrinsic CZM.

When considered separately, continuous and discontinuous approaches are unable to model with high accuracy the fracture process of ductile materials. This process within a material often begins with a global interaction of all the pre-existing microscopic defects followed by their growth in strain concentration areas, until the coalescence of some defects creates a macroscopic crack. On the one hand, this cannot be modeled by the sole recourse to a discontinuous approach such as the CZM since the degradation process cannot be modeled with the same accuracy as with a CDM. On the other hand, a CDM can capture the damage diffusion stage, the damage evolution in a process zone, and finally the localization of the damage. However, without introducing a crack, large element distortions arise as a result of the stress-carrying capacity loss of the constitutive material response. These large element distortions not only disagree with the physical reality but also hurt the numerical convergence of the simulations. Moreover, because of the non-local or gradient enhanced formulation, the excessive strain on the fictitious crack surface leads to an unrealistic extension of the damage field. These problems motivated the introduction of a crack when using a non-local CDM, either by remeshing techniques when the damage is close to one $[28,29]$, or by having recourse to an xFEM scheme [30, 31, 32], but with a loss of energy. The remeshing technique or a method implying a loss of energy can remain accurate when the crack introduction physically occurs for damage values close to one, but this is not necessarily the case for ductile materials for which the failure was observed experimentally for lower values of the damage. Finally the crack could also be introduced in a local CDM using an EFEM upon loss of ellipticity [33] or using the thick level set approach [34] in which case the damage becomes non-local in the sense of being averaged over a certain thickness in the wake of the front.

Despite these achievements, a practical and efficient implementation of the damage to crack transition problem is still challenging. The objective of this paper is to establish an efficient numerical framework for fracture simulations in which the transition from damage to crack is carried out based on the preservation of dissipated energies. The implicit damage model $[5,6,7,8]$ is chosen as the continuous description since it can simulate the damage evolution and strain softening phenomena. The CZM is integrated in the hybrid DG/ECL to model the discontinuous stage. Finally, upon damage to crack transition, the TSL of the CZM is defined to respect the equivalence between the fracture energy of the non-local damage and cohesive crack following the work in $[35,36,37]$. Note that this theoretical framework has already been used for brittle materials to define the transition from a non-local implicit CDM to a CZM embedded in the XFEM method [38] and the transition from a gradient enhanced damage model to an intrinsic cohesive crack in a predefined 
direction (mode I) [39].

The implementation of the method is made efficient because of the choice of the hybrid DG/ECL to integrate the CZM. Indeed, the method offers many advantages: (i) The method can efficiently be integrated in a parallel FE code and remains scalable for a high number of processors (a few thousands [23]); (ii) The method does not require complex modifications of the $\mathrm{FE}$ code (no modification of the volume elements required); (iii) As the non-local damage-enhanced elasto-plastic material behavior is fully resolved at the interface, it is possible to define a damage to crack criterion based on the stress, damage or pressure fields, allowing multiple crack initiation/propagation to occur. However the DG approach suffers from a higher number of degrees of freedom, which is strengthened by the fine mesh required by the use of CZM. Indeed as the crack has to follow the element boundary, a fine mesh is required to ensure convergence of the crack path and dissipation energy, at least for unstructured meshes [40, 41]. However a fine mesh is always required to capture complex crack propagation, whatever the discretization technique used, and in the particular case of the DG method this drawback is mitigated by the high scalability of the method.

The paper is organized as follows. In Section 2 the theoretical formalism to construct a TSL from a CDM when a transition has been decided is developed based on the existing theoretical background of energetic equivalence between the two concepts $[35,36,37]$. We also propose a transition criterion based on the effective stress state, which avoids elements blow-up during the numerical simulation. The consistent and computationally efficient implementation of the non-local implicit CDM-CZM hybrid method is developed within a DG framework in Section 3. A staggered explicit-direct resolution scheme [29] of the finite element model which comprise displacement and non-local degrees of freedom is then proposed in a parallel setting in Section 4. Section 5 illustrates the efficiency of the hybrid non-local implicit DG/ extrinsic cohesive law framework on two standard problems. First the effect of the damage to crack transition criterion is investigated on a compact tension specimen (CTS). It is shown that the introduction of the crack does not modify the loading part of the structural response. Then, the effect of the mesh size is studied by considering the double-notched specimen (DNS) test which is characterized by the initiation of a crack at the two notches. These initiations are well captured by the method and the structural response is found to be insensitive to the mesh size.

\section{Non-local damage continuum to cohesive discontinuity transition model}

In this section, the construction of an energetically consistent TSL when one decides to shift from CDM to a CZM is studied. First the theoretical background of both the CZM and the implicit non-local CDM are briefly summarized. Then the main results developed in $[35,36,37]$ to derive the equivalence between the energy dissipated with both models are given. Based on this we construct a TSL in the particular case of the scalar elastic damage model expressed in a 1D implicit gradient setting. The application to the 3D case and the transition criterion are finally developed in this section.

\subsection{Constitutive models}

The theoretical backgrounds of the cohesive model and of the non-local continuum damage model are herein briefly recalled. In particular, emphasis is put on their energetic aspects to introduce the consistent transition scheme.

\subsubsection{Cohesive zone model}

The cohesive zone model (CZM) was pioneered by Barenblatt [10] and Dugdale [11] in fracture models to account for the traction evolution between crack lips during the separation process. During this crack opening process, the traction $\bar{t}$ decreases monotonically from the material strength $\sigma_{\mathrm{C}}$ until reaching zero at a critical opening $\Delta_{\mathrm{C}}$ to model a progressive irreversible damage of the material, see Fig. 1(a). If unloading happens during this damaging stage the TSL becomes reversible, with a reduced elastic stiffness associated to the damage evolution. 

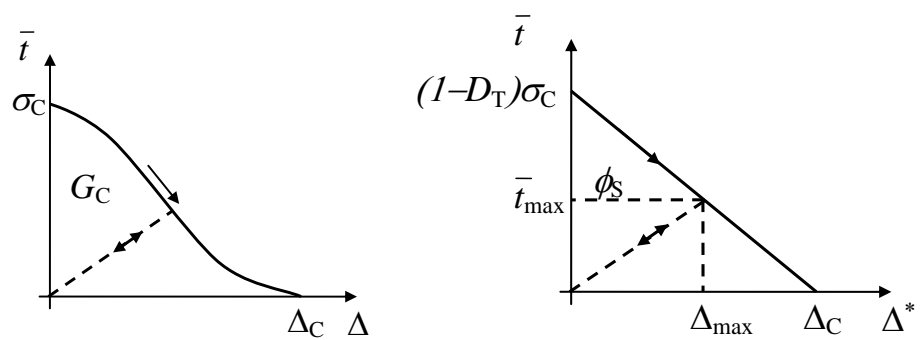

Figure 1: Extrinsic cohesive laws: (a) General extrinsic cohesive law characterized by the strength $\sigma_{\mathrm{C}}$, the critical opening $\Delta_{\mathrm{C}}$ and the critical energy release rate $G_{\mathrm{C}}$. (b) Construction of a linear extrinsic TSL at the CDM-CZM shift: traction $\bar{t}$ in terms of the effective opening $\Delta^{*}, D_{\mathrm{T}}, \sigma_{\mathrm{C}}=\frac{\sigma_{\mathrm{T}}}{1-D_{\mathrm{T}}}, \Delta_{C}^{*}$ and $\phi_{\mathrm{S}}=\left(1-D_{\mathrm{T}}\right) \frac{\Delta_{\mathrm{C}}^{*} \sigma_{\mathrm{C}}}{2}$ are respectively the damage at shift, the strength, the critical effective opening, and the remaining energy to be dissipated.

The energy dissipated during the irreversible process corresponds to the fracture energy $G_{\mathrm{C}}$ per unit crack surface, which can be expressed as

$$
G_{\mathrm{C}}=\int_{0}^{\Delta_{\mathrm{C}}} \bar{t} \mathrm{~d} \Delta
$$

In a finite element context, the surface separation $\Delta$ can be obtained from the discontinuity of the displacement field $\llbracket \boldsymbol{u} \rrbracket$. The increment of energy dissipation resulting from a crack propagation is given by Griffiths theory

$$
\mathrm{d} \Phi_{\mathrm{S}}=G_{\mathrm{C}} \mathrm{d} A,
$$

where $A$ is the area of fully opened crack.

\subsubsection{Implicit non-local damage model}

Let us consider an isothermal elastic case for simplicity. Following the thermodynamical formulation of the CDM proposed in [42], the free (reversible) energy stored per unit volume, $\rho \psi$, of the damaged material is defined in terms of the elastic deformations $\varepsilon$ as

$$
\rho \psi=\frac{1}{2} \varepsilon: \mathbb{C}^{\mathrm{D}}(D): \varepsilon,
$$

where $\mathbb{C}^{\mathrm{D}}(D)$ is the equivalent secant fourth-order tensor of the material response evolving with the damage value $D$. The stress tensor $\boldsymbol{\sigma}$ is calculated from $\psi$ as

$$
\boldsymbol{\sigma}=\rho \frac{\partial \psi}{\partial \varepsilon}=\mathbb{C}^{\mathrm{D}}: \varepsilon
$$

and the damage energy release rate $Y$ is defined by

$$
Y=-\rho \frac{\partial \psi}{\partial D}=-\frac{1}{2} \varepsilon: \frac{\partial \mathbb{C}^{\mathrm{D}}}{\partial D}: \varepsilon \geq 0 .
$$

The Clausius Duhem inequality constrains the increment of energy dissipated to be positive following

$$
d \phi=\boldsymbol{\sigma}: d \boldsymbol{\varepsilon}-\rho d \psi \geq 0 .
$$

From Eqs. (3-5), one has $d \psi=-Y d D+\sigma: d \varepsilon$ and this last equation becomes

$$
d \phi=Y d D \geq 0,
$$

where $d D \geq 0$ is the increment of damage. Therefore, in a structure of volume $V$, the increment of dissipated energy can be expressed as

$$
\mathrm{d} \Phi=\int_{V} d \phi \mathrm{d} V=\int_{V}(Y \mathrm{~d} D) \mathrm{d} V .
$$


The damage parameter $D$ evolves according to the deformation history of the material while satisfying a loading surface function of the strain state, in the local form of CDM theory, which reads $f\left(\varepsilon, \mathbb{C}^{\mathrm{D}}, \kappa_{\mathrm{i}}\right) \leq 0$, where $\kappa_{\mathrm{i}}$ is an initial threshold. The damage evolution is governed by a function $\mathcal{F}$ of the strain, still in the local form, following

$$
d D= \begin{cases}0 & \text { if } f<0 \text { or }(f=0 \text { and } d f<0) \\ \mathcal{F}(\varepsilon) & \text { if } f=0 \text { and } d f=0 .\end{cases}
$$

In a non-local formulation, the loading surface is expressed in terms of a non-local strain $\tilde{\varepsilon}$, and reads $f\left(\tilde{\varepsilon}, \mathbb{C}^{\mathrm{D}}, \kappa_{0}\right) \leq 0$, while the damage evolution is now governed by a function $\mathcal{F}$ of the non-local strain, following

$$
d D= \begin{cases}0 & \text { if } f<0 \text { or }(f=0 \text { and } d f<0) \\ \mathcal{F}(\tilde{\varepsilon}) & \text { if } f=0 \text { and } d f=0 .\end{cases}
$$

These expressions depend on the non-local model considered and on the material response characterized by a damage model. In this paper we consider the implicit non-local formulation developed in [5, 6] for a scalar damage model under the assumption of isotropic damage. Although this model remains simplistic it has been widely studied in the literature, including to consider the damage to crack transition [36, 37], and calibrated experimentally for short glass-fiber reinforced polymers [6], which allows the application presented later on to be validated. In a future work it is intended to consider more complex (elasto-plastic) damage models.

In the scalar damage context $\mathbb{C}^{\mathrm{D}}(D)$ can be expressed as $(1-D) \mathbb{C}$, where $\mathbb{C}$ is the initial elastic tensor of the virgin material. The effective Cauchy stress $\hat{\boldsymbol{\sigma}}$ concept [2] is thus recovered from (4) with

$$
\boldsymbol{\sigma}=(1-D) \hat{\boldsymbol{\sigma}}=(1-D) \mathbb{C}: \boldsymbol{\varepsilon} .
$$

The loading function is now expressed as

$$
f\left(\tilde{e}, D, \kappa_{\mathrm{i}}\right)=\tilde{e}-\kappa(D) .
$$

In this function $\tilde{e}$ is a non-local equivalent strain, and $\kappa(D)$ is a monotonically increasing deformation history parameter which is initially set to $\kappa_{\mathrm{i}}$, with

$$
\kappa(t)=\max \left[\kappa_{\mathrm{i}}, \tilde{e}(\varepsilon, \tau) \mid \tau \leq t\right],
$$

where $t$ is the history parameter. The damage evolution (10) was approximated, by integration, for short glass-fiber reinforced polymers in [43] by

$$
D=1-\left[\frac{\kappa_{\mathrm{i}}}{\kappa}\right]^{\beta}\left(\frac{\kappa_{\mathrm{C}}-\kappa}{\kappa_{\mathrm{C}}-\kappa_{\mathrm{i}}}\right)^{\alpha},
$$

where $\kappa_{\mathrm{i}}$ is the initial value of $\kappa$ triggering the damage evolution, the critical historical parameter $\kappa_{\mathrm{C}}$ defines the value of $\kappa$ for which the damage $D$ reaches its ultimate value 1 , and where the other material coefficients are $\alpha$ and $\beta$.

The equivalent strain $e$ is evaluated from the positive principle strain components $\varepsilon_{i}^{+}[42]$

$$
e=\sqrt{\sum_{i=1,2,3}\left(\varepsilon_{i}^{+}\right)^{2}}
$$

while the non-local equivalent strain $\tilde{e}$ results from the resolution of the Helmholtz-type equation:

$$
\tilde{e}-c \nabla^{2} \tilde{e}=e .
$$

In this equation $\sqrt{c}$ is the characteristic size of the material. The governing Eq. (16) is completed be the natural boundary conditions $(\mathrm{BC})$

$$
\frac{\partial \tilde{e}}{\partial \boldsymbol{n}}=n_{i} \frac{\partial \tilde{e}}{\partial n_{i}}=0
$$


with $\boldsymbol{n}$ the outward unit normal. This gradient enhanced formula is actually derived from the non-local integral approach

$$
\tilde{e}(\boldsymbol{x})=\int_{V_{\mathrm{C}}} W(\boldsymbol{y} ; \boldsymbol{x}) e(\boldsymbol{y}) \mathrm{d} V,
$$

with a particular choice of the weight function $W(\boldsymbol{y} ; \boldsymbol{x})$, i.e. the Green function $W_{\mathrm{G}}$, which can be written as $[44]$

$$
W_{\mathrm{G}}(\boldsymbol{x} ; \boldsymbol{y})=\frac{1}{2 \sqrt{c}} \exp \left[-\frac{|\boldsymbol{x}-\boldsymbol{y}|}{\sqrt{c}}\right]+W_{\mathrm{G}}^{\mathrm{BC}}(\boldsymbol{x} ; \boldsymbol{y}),
$$

where the second term has to be particularized with the problem to satisfy the BC (17), see Section 2.2.3 for a $1 \mathrm{D}$ application.

Finally, as suggested by Geers et al. [43], instead of using a constant $c$, a variable $c(e)$ is considered to avoid artificial spreading of the damage zone orthogonality to the direction of crack propagation. The variable $c(e)$ reads

$$
c(e)=\left\{\begin{array}{ll}
c_{0}\left(\frac{e}{e_{\mathrm{C}}}\right)^{n_{\mathrm{C}}} & \text { if } \quad e \leq e_{\mathrm{C}} \\
c_{0} & \text { if } \quad e>e_{\mathrm{C}}
\end{array},\right.
$$

which is referred to as the gradient activity in their work. ${ }^{1}$

Note that an anisotropic version of the non-local implicit equations (16) and (17) exists for composite materials, e.g. [45].

\subsection{Energetic equivalence between $C D M$ and $C Z M$}

In this part, the main results developed in $[35,36,37]$ are first recalled in a general case, before being particularized to a $1 \mathrm{D}$ problem. This $1 \mathrm{D}$ solution is then exploited to construct the TSL upon transition from a CDM to a CZM providing this transition occurs after the strain softening onset. Finally the particular case of an exponential non-local damage model is treated for illustration purpose.

\subsubsection{Theoretical background}

Let us first analyze the similarities of the two approaches for fracture problems. CDM uses damage areas to simulate the degradation of materials and cracks are usually represented by fully damaged $($ e.g. $D=1.0)$ zones. The CZM uses fictitious cracks to reflect locally damaged materials until the total opening of the crack is achieved, which happens when the traction $\bar{t}$ between the crack surfaces vanishes. Both methods describe the progressive loss of stress carrying capacity of the material in the process zone. During this process, the equivalence of the two models must be thermodynamically acceptable.

Since the energy equivalence requires the two increments of dissipated energy for the CZM $d \Phi_{S}(2)$ and for the $\mathrm{CDM} d \Phi(8)$ to be equal, we get the following relation for crack propagation:

$$
\mathrm{d} A=\frac{\int_{V}(Y \mathrm{~d} D) \mathrm{d} V}{G_{\mathrm{C}}}
$$

However, the CZM defines the initiation of crack as soon as the traction $\bar{t}$ reaches the material strength $\sigma_{\mathrm{C}}$, after which the process enters the crack propagation stage. Hence, the cohesive zone model can only reflect the energy dissipated during the crack propagation, which means that Eq. (21) only holds for crack propagation, while with a CDM the crack initiation and propagation are all governed by the damage evolution without explicit separation of these two cracking stages. Therefore, in order to derive the TSL of the cohesive model equivalent to the damage model, the energy dissipation during crack initiation and propagation needs to be separated in the damage model.

\footnotetext{
${ }^{1}$ This model leads to one more freedom on each node in its finite element implementation. Here to simplify the implementation and to reduce the system size, we use the local equivalent strain obtained at the previous time step to calculate the characteristic length, say $c=c\left(e_{n}\right)$ during the time interval $\left[t_{n}, t_{n+1}\right]$ of the simulation.
} 
In the work of Cazes et al. [37], the total energy dissipated during the damaging process, $\Phi$, is divided into two parts, the volume dissipation $\Phi_{\mathrm{vol}}$ and the localized dissipation $\Phi_{\mathrm{loc}}$,

$$
\Phi=\Phi_{\mathrm{vol}}+\Phi_{\mathrm{loc}} .
$$

The volume dissipation $\Phi_{\mathrm{vol}}$ refers to the situation prior to localization, when the only existing damage is the diffuse damage. After localization, the localized damage zone tends to unload the material in its vicinity and the energy dissipation comes from the damage zone only. Thus $\mathrm{d} \Phi_{\mathrm{vol}}=0$ and we have

$$
\mathrm{d} \Phi_{\mathrm{S}}=\mathrm{d} \Phi_{\text {loc }}=\mathrm{d} \Phi .
$$

This relation linking the increments of dissipated energy can be used to construct the TSL of the CZM from the knowledge of the damage model. Let us assume a transition from the CDM to the CZM happening for a damage value $D_{\mathrm{T}}$, after localization has occurred. In that case, the energy that has to be dissipated through the TSL satisfies

$$
\Phi_{\mathrm{S}}=\Phi-\Phi_{\mathrm{vol}}-\Phi_{\mathrm{DZ}}=\Phi_{\mathrm{loc}}-\Phi_{\mathrm{DZ}},
$$

where $\Phi_{\mathrm{DZ}}$ is the energy already dissipated due to the CDM model in the damaged zone when $D \leq D_{\mathrm{T}}$. In this description a regularized damage model, as the implicit non-local model, must be used to obtain a reliable energy dissipation during damaging process without mesh-dependence.

These energy dissipation relations between damage and cohesive zone models are now studied in the simple 1D setting of a bar under tension.

\subsubsection{One-dimensional setting}

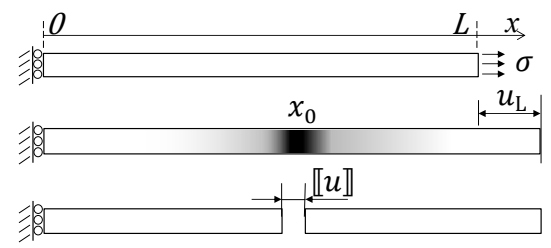

(a) Comparison of CDM and CZM in a 1D

bar

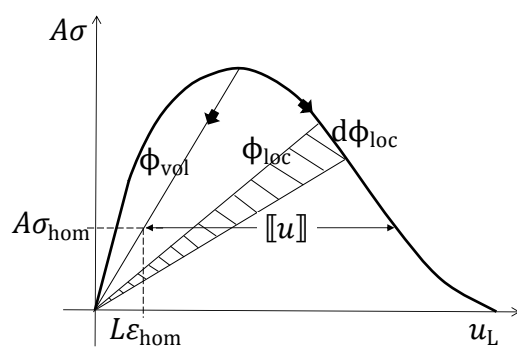

(b) Force-elongation curve with $\mathrm{CDM}$

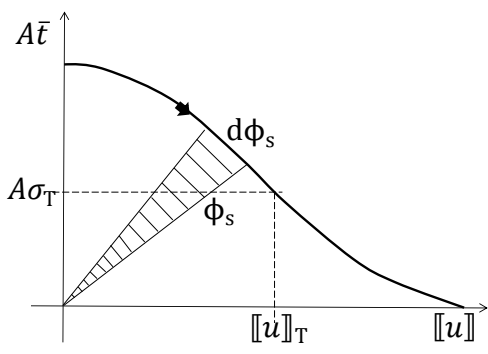

(c) CZM constructed from the CDM

Figure 2: Equivalence between the CZM and the CDM just after localization has occurred. (a) 1D bar of cross-section $A$ and with the strain localizing at $x_{0}$. (b-c) Construction of the full CZM TSL from the force-elongation curve obtained with the $\mathrm{CDM}$. The total localization energy, $\Phi_{\text {loc }}$, of the damage law is the surface area of the force-displacement curve beyond strain softening and should correspond to the dissipation energy of the TSL, $\Phi_{\mathrm{S}}$.

A bar of cross-section $A$ is subjected to a traction loading $A \sigma$. The length of the bar is $L$ and the strain localization is assumed to start at a predefined point $x_{0}$, see Fig. 2(a). A CZM can be constructed to be equivalent to the CDM, once the strain softening onset is reached, meaning that the damage law could be substituted by a CZM at the strain softening onset when the damage localization zone has just been formed. 
Let us first consider the continuum elastic model. Using Eqs. (3-4), the free energy of the bar pictured in Fig. 2(a) can be written as

$$
\Psi=\int_{V} \rho \psi d V=\frac{A}{2} \int_{0}^{L} \sigma \varepsilon \mathrm{d} x .
$$

Assuming an isothermal process, the energy dissipation increment for the CDM can be calculated from

$$
\mathrm{d} \Phi=\mathrm{d} W_{\text {int }}-\mathrm{d} \Psi=\frac{A}{2} \int_{0}^{L}(\sigma \mathrm{d} \varepsilon-\varepsilon \mathrm{d} \sigma) \mathrm{d} x,
$$

where $W_{\text {int }}$ is the work of internal forces of the whole structure, see [37] for details.

The corresponding discontinuous configuration is a bar of homogeneous strain $\varepsilon_{\text {hom }}$ with a displacement jump $\llbracket u \rrbracket$ at $x=x_{0}$, see Fig. 2(a). In this one-dimensional case, the compatibility of the displacement leads to

$$
\llbracket u \rrbracket=\int_{0}^{L} \varepsilon \mathrm{d} x-\int_{0}^{L} \varepsilon_{\text {hom }} \mathrm{d} x,
$$

as shown in Fig. 2(a). The free energy $\Psi$ of this discontinuous configuration can be calculated from two parts: the free energy associated to the surface discontinuity [37]

$$
\Psi_{\mathrm{S}}=\frac{1}{2} A \bar{t} \llbracket u \rrbracket
$$

and the free energy due to the strain over the bar

$$
\Psi_{\text {vol }}=\frac{A}{2} \int_{0}^{L} \sigma \varepsilon_{\text {hom }} \mathrm{d} x=\frac{A \bar{t}}{2} \int_{0}^{L} \varepsilon_{\text {hom }} \mathrm{d} x,
$$

as the strain is uniform in the bar. During the strain softening stage the energy dissipation only results from the surface discontinuity, which yields using (28)

$$
\mathrm{d} \Phi_{\mathrm{S}}=A \bar{t} \mathrm{~d} \llbracket u \rrbracket-\mathrm{d} \Psi_{\mathrm{S}}=\frac{A}{2}(\bar{t} \mathrm{~d} \llbracket u \rrbracket-\llbracket u \rrbracket \mathrm{d} \bar{t}),
$$

see [37] for details.

In order to compare (26) to (30) in the $1 \mathrm{D}$ case of uniform $\sigma$, the differentiation of Eq. (27) can be introduced in Eq. (26). Beyond strain localization, there is an elastic unloading over the continuous volume of the bar, see Fig. 2(b), and (26) becomes [37]

$$
\left.\mathrm{d} \Phi=\frac{A}{2}(\sigma \mathrm{d} \llbracket u \rrbracket)-\llbracket u \rrbracket \mathrm{d} \sigma\right) .
$$

Comparing (31) to (30), we verify $\mathrm{d} \Phi=\mathrm{d} \Phi_{\mathrm{S}}$, see Figs. $2(\mathrm{~b})$ and $2(\mathrm{c})$, when $\sigma=\bar{t}$. For this onedimensional case, it appears that to construct a cohesive law from the non-local damage model, the traction $\bar{t}$ should be equal to the uniform tension $\sigma$ in the bar and the surface separation should be computed from Eq. (27).

\subsubsection{Construction of the TSL upon transition from a CDM to a CZM in a one-dimensional setting}

Let us consider a bar in tension, see Fig. 3(a). For a practical application of the CZM the parameters which allow defining the TSL are the total dissipated energy $\Phi_{\mathrm{S}}$ and the maximum tension $\max (\bar{t})$ at the starting point of the cohesive law. Thus it becomes straightforward to construct a cohesive law when we decide to shift from the CDM to CZM at a given damage value, such as $D_{\mathrm{T}}$, if this value is beyond the strain softening onset. Indeed, on the one hand the maximum tension $\max (\bar{t})$ is directly obtained from the stress $\sigma_{\mathrm{T}}$ reached at $D=D_{\mathrm{T}}$, see Figs. 3(b) and 3(c), and on the other hand the total dissipation energy $\Phi_{\mathrm{S}}$ characterizing the TSL, see Fig. 3(c), is computed using Eq. (24) from the force-displacement curve of the full damage model. In the $1 D$-setting, the total localization energy, $\Phi_{\text {loc }}$, of the damage law is depicted 


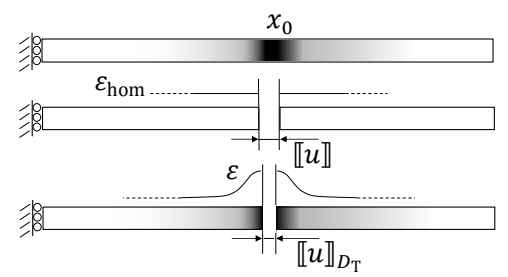

(a) Comparison of CDM and CZM in a 1D bar

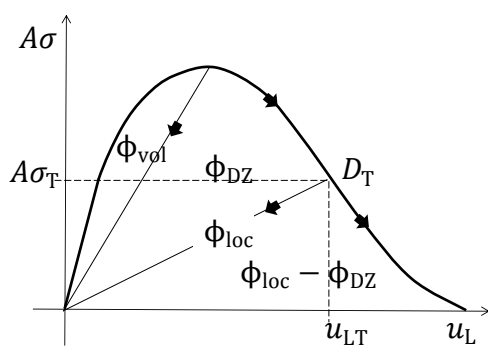

(b) Force-elongation curve with CDM, definition of a transition damage $D_{\mathrm{T}}$

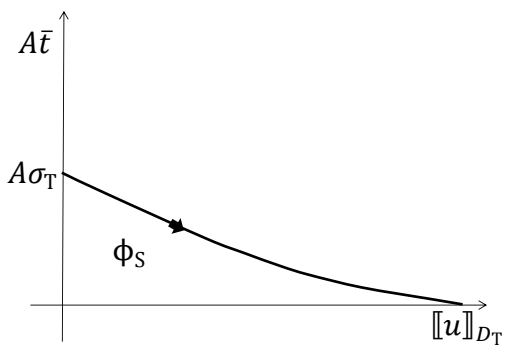

(c) CZM after transition from CDM at $D_{\mathrm{T}}$

Figure 3: Equivalence between a CZM and the CDM for a transition happening during the strain softening. (a) 1D bar of cross-section $A$ and with the strain localizing at $x_{0}$. After strain softening onset the strain is no longer homogeneous in the bar and the opening $\llbracket u \rrbracket_{D_{T}}$ is different from the opening $\llbracket u \rrbracket$ defined in Eq. (27) for a homogeneous strain. (b-c) Construction of the remaining TSL after transition from a CDM. At the transition point the damage has reached a value $D_{\mathrm{T}}$. The total localization energy, $\Phi_{\text {loc }}$, of the damage law is the surface area of the force-displacement curve beyond strain softening, the energy actually dissipated at transition, $\Phi_{\mathrm{DZ}}$, is the surface area of the force-displacement curve between the strain softening and the damage $D_{\mathrm{T}}$, and the difference $\Phi_{\mathrm{loc}}-\Phi_{\mathrm{DZ}}$ should correspond to the energy dissipation, $\Phi_{\mathrm{S}}$, of the TSL.

on Fig. 3(b) as the surface area of the force-displacement curve beyond strain softening, and the energy actually dissipated, $\Phi_{\mathrm{DZ}}$, is depicted on Fig. $3(\mathrm{~b})$ as the surface area of the force-displacement curve between the strain softening and the damage value $D_{\mathrm{T}}$.

However, contrarily to the case for which the CZM substitutes the CDM at strain softening onset, the surface separation $\llbracket u \rrbracket_{D_{\mathrm{T}}}$ cannot be directly calculated from Eq. (27). Indeed, the strain in the bar is not homogeneous anymore as the parts of the bar beside the discontinuous surface are in the localizing damage zone and are thus subjected to a higher damage, see Fig. 3(a). As the stress is uniform in the bar, the strain in the localization neighborhood is higher. Thus the energy equivalence between the damage model and the cohesive zone model developed in Section 2.2.2 has to be used to compute the total dissipation energy $\Phi_{\mathrm{S}}$. Considering a shift from a CDM to a CZM for a damage reaching a value $D_{\mathrm{T}}$, assuming the shift has actually occurred at the strain softening onset this would correspond to an opening $\llbracket u \rrbracket_{\mathrm{T}}$ of the equivalent TSL computed at Section 2.2.2, see Fig. 2(c). The energy $\Phi_{\mathrm{S}}$ that remains to be dissipated can then be integrated from this fictitious case, see Fig. 2(c), and reads:

$$
\Phi_{\mathrm{S}}=\frac{A}{2} \sigma_{\mathrm{T}} \llbracket u \rrbracket_{\mathrm{T}}+A \int_{\llbracket u \rrbracket_{\mathrm{T}}}^{\infty} \bar{t} \mathrm{~d} \llbracket u \rrbracket .
$$

This value of the energy can now be reported in the new TSL in terms of the real opening $\llbracket u \rrbracket_{D_{\mathrm{T}}}$, see Fig. $3(\mathrm{c})$.

\subsubsection{Application to the case of an exponential non-local damage law}

In this section, the problem of calculating the TSL of a cohesive law from a damage model is solved by particularizing and resolving the equations derived in Section 2.2.3 to the case of an exponential non-local elastic damage model (14) developed in Section 2.1.2. 
Description of the problem. The most natural way to obtain a cohesive law is to compute a loadingdisplacement curve following a damage model, as presented in Fig. 2(b). We need to mention that in the case of snap-back, the jump of displacement can still be computed through Eq. (27).

Here we use the one-dimensional bar illustrated in Fig. 2(a), characterized by a strain localization initiated at $x=x_{0}$. Therefore, the strain localization zone or damage localization zone develops around $x_{0}$, while the strain is homogeneous outside of this zone. Considering the implicit gradient enhanced damage model presented in Section 2.1.2, the equivalent strain $e$ corresponds to the strain $\varepsilon(x)$, and the non-local equivalent strain $\tilde{e}$ evolution is governed by Eq. (16) or by Eq. (18). In order to solve Eq. (18), the weight function (19) needs to be completed to satisfy the boundary conditions (17), which read for the 1D bar problem

$$
\frac{\mathrm{d} e}{\mathrm{~d} x}=0 \quad \text { at } \quad x=0 \quad \text { and } \quad x=L .
$$

The complete weight function for the 1D bar are thus expressed as [44]

$$
W(x ; y)=\frac{1}{2 \sqrt{c}} \exp \left[-\frac{|x-y|}{\sqrt{c}}\right]+\frac{A(y)}{2 \sqrt{c}} \exp \left[\frac{x}{\sqrt{c}}\right]+\frac{B(y)}{2 \sqrt{c}} \exp \left[\frac{L-x}{\sqrt{c}}\right],
$$

where

$$
\begin{aligned}
A(y) & =\frac{\exp \left[\frac{y}{\sqrt{c}}\right]+\exp \left[-\frac{y}{\sqrt{c}}\right]}{\exp \left[\frac{2 L}{\sqrt{c}}\right]-1}, \\
B(y) & =\frac{\exp \left[\frac{L-y}{\sqrt{c}}\right]+\exp \left[-\frac{L-y}{\sqrt{c}}\right]}{\exp \left[\frac{2 L}{\sqrt{c}}\right]-1} .
\end{aligned}
$$

The problem is thus restated as follows

- A one dimensional bar of cross-section $A$ is subjected to a tensile load $A \sigma$, see Fig. 2(a), and the displacement field satisfies

$$
u(0)=0 \quad \text { and } \quad u(L)=u_{L},
$$

where $L$ is the length of the bar. The damage zone localizes around $x=x_{0}$, the local equivalent strain and non-local equivalent strain in this damage localization zone read

$$
e(x)=\varepsilon(x) \quad \text { and } \quad \tilde{e}(x)=\int_{0}^{L} W(y ; x) e(y) \mathrm{d} y,
$$

where $W(y ; x)$ is given by Eq. (34). Since the strain is homogeneous out of the damage localization zone, the local and non-local equivalent strains are homogeneous too, and out of this localization zone we have

$$
\tilde{e}_{\mathrm{hom}}=e_{\mathrm{hom}}=\varepsilon_{\mathrm{hom}} .
$$

- Considering the damage model presented in Section 2.1.2, we call

$$
D_{\text {hom }}=D\left(\kappa_{\text {hom }}\right) \quad \text { where } \quad \kappa_{\text {hom }}=\max \left[\kappa_{\mathrm{i}}, \tilde{e}_{\text {hom }}(\tau) \mid \tau \leq t\right]
$$

the diffuse homogeneous damage at current time $t$ out of the localization zone, and we call

$$
D(x)=D(\kappa(x)) \quad \text { where } \quad \kappa(x)=\max \left[\kappa_{\mathrm{i}}, \tilde{e}(x, \tau) \mid \tau \leq t\right]
$$

the damage distribution in the localization zone. In the $1 \mathrm{D}$ setting, the stress is uniform and reads

$$
\sigma=(1-D(x)) E \varepsilon(x)=\left(1-D_{\text {hom }}\right) E \varepsilon_{\text {hom }},
$$

where $E$ is the (undamaged) elastic modulus of the bar. 
- The problem can thus be stated as finding, during the damage evolution $D\left(x_{0}\right)$ from 0 to 1 , the relation

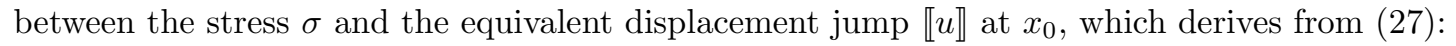

$$
\llbracket u \rrbracket=\int_{0}^{L} \varepsilon(x) \mathrm{d} x-\int_{0}^{L} \varepsilon_{\mathrm{hom}} \mathrm{d} x=u_{L}-L \varepsilon_{\mathrm{hom}} .
$$

It is rather difficult to obtain an analytical solution for the implicit gradient enhanced damage problem, even for the one-dimensional case represented by Eqs. (36-41). Peerlings [5] has derived a semi-analytical solution in the particular case of a damage law equivalent to perfect plasticity, i.e. when a damage growth results in a constant stress, but for more complicate material behaviors a numerical solution is required.

An approximate analytical solution for the one dimensional setting. In this paper, to avoid the burden of resolving a numerical problem we consider an approximate resolution, which allows deriving analytically the TSL from the damage evolution model.

Toward this end, we assume an analytical non-local equivalent strain field $\tilde{e}$ deduced from a reference displacement field $u(x)$, with a discontinuity $\llbracket u \rrbracket$ at the point $x_{0}$. Since this assumed field $u(x)$ needs to be thermodynamically admissible compared to the displacement field of the non-local damage model, a straightforward choice of $u(x)$ to evaluate the equivalent non-local strain field is to use the displacement discontinuity of the cohesive zone model embedded in a the homogeneous strain field:

$$
u(x)=\varepsilon_{\mathrm{hom}} x+\llbracket u \rrbracket H\left(x-x_{0}\right),
$$

which satisfies (42) and where $H\left(x-x_{0}\right)$ is the Heaviside function. The corresponding strain field over $[0, L]$ reads

$$
\varepsilon(x)=\varepsilon_{\mathrm{hom}}+\llbracket u \rrbracket \delta\left(x-x_{0}\right),
$$

with $\delta$ being the Dirac function. Using Eq. (34) and (37), the non-local equivalent strain field $\tilde{e}$ reads

$$
\tilde{e}(x)=\int_{0}^{L} W(y ; x)\left[\varepsilon_{\mathrm{hom}}+\llbracket u \rrbracket \delta\left(y-x_{0}\right)\right] \mathrm{d} y=\varepsilon_{\mathrm{hom}}+\llbracket u \rrbracket W\left(x_{0} ; x\right) .
$$

According to the comparison of results provided by Dufour et al. [46] for a one-dimensional case, the distribution of the non-local equivalent strain derived from a strong discontinuous approach, as presented by Eq. (45), matches within a few percents error the results obtained by solving the strict non-local continuous problem, if the damage is rather high. Hence, we will use this estimation (45) to obtain the equivalent cohesive law of the non-local model and later to define the transition from a continuous CDM to a discontinuous CZM.

Now let us consider the Eqs. (41) and (42). The damage $D_{\text {hom }}$ represents the maximum diffuse damage, which is a constant value during the strain softening stage. Indeed, for a material characterized by given parameters of its constitutive and damage models, $D_{\text {hom }}$ corresponds to the damage value reached at the onset of stain softening, and is thus considered as a known variable. The value of $D_{\text {hom }}$ in the system of Eqs. (39-42) is thus a known value during the strain softening stage. Note that the damage in the damage zone keeps increasing during the strain softening stage.

With this approximate model, for a given $\varepsilon_{\text {hom }}$, which is a rather low value during the strain softening stage as the localized damage values are high, the corresponding displacement jump $u \rrbracket$ can be calculated from the set of Eqs. (39-42) completed by Eq. (45). Therefore, the cohesive law $\bar{t}$ vs. $\Delta$, which corresponds to the relation $\sigma$ vs. $\llbracket u \rrbracket$ in the $1 \mathrm{D}$ case, can be constructed for high damage stages.

Application example. As an illustration example we consider the short glass-fiber-reinforced polypropylene material whose properties are given in the benchmark study in [43]. The considered material has a Young modulus $E$ of $3.2 \mathrm{GPa}$, and follows a power damage evolution law (14), with $\kappa_{\mathrm{i}}=0.011, \kappa_{\mathrm{C}}=0.5, \alpha=5.0$, and with $\beta=0.75$. When damage is high, the characteristic length of this material is $\sqrt{2} \mathrm{~mm}$ according to the reference [43]. Fig. 4(a) shows the material response in tension without strain localization effect. 


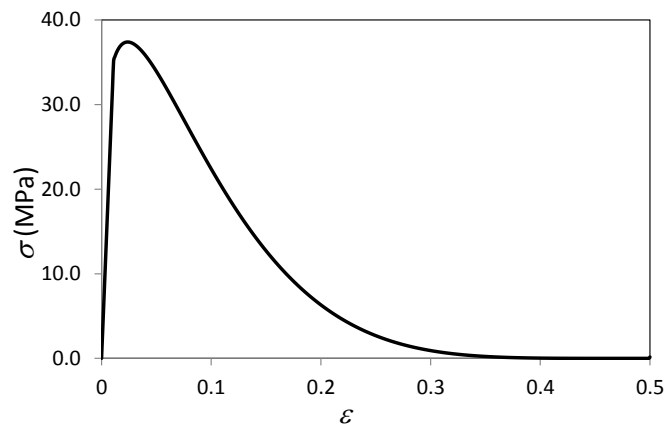

(a) $\sigma$ vs. $\varepsilon$

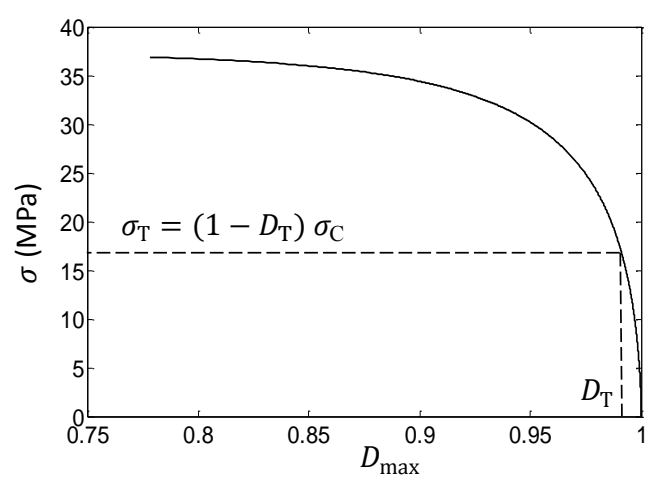

(c) $\sigma$ vs. $D_{\max }$

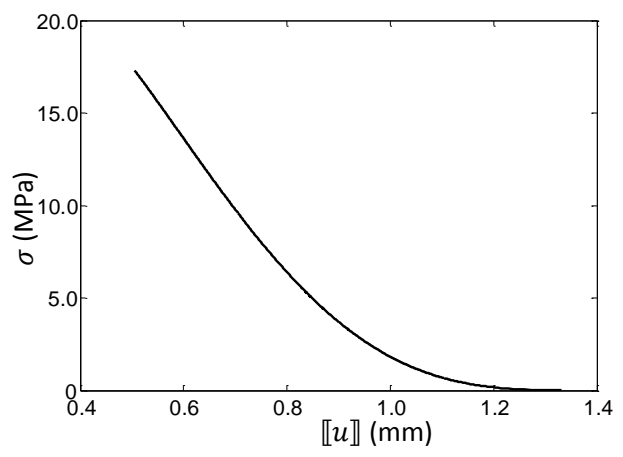

(b) $\sigma$ vs. $\llbracket u \rrbracket$

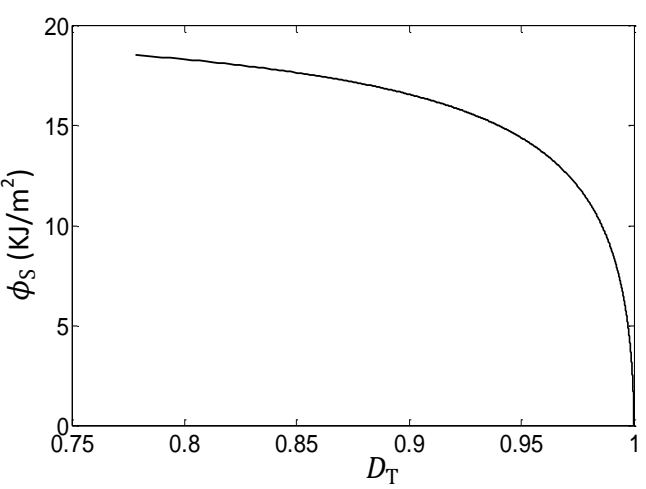

(d) $\phi_{\mathrm{S}}$ vs. $D_{\mathrm{T}}$

Figure 4: Approximate resolution of the CDM-TSL transition for short glass-fiber-reinforced polypropylene. (a) Tensile stressstrain relation for the short glass-fiber-reinforced polypropylene, the strain localization is not considered. (b) The relation between the traction and displacement jump for an equivalence between the CDM and CZM; (c) Stress in the bar in term of the maximum value of damage $D_{\max }$ at $x_{0}$. Assuming a transition from CDM to CZM for a given value of damage $D_{\mathrm{T}}$ reached at $x_{0}$, the curve $\sigma_{\mathrm{T}}$ vs. $D_{\mathrm{T}}$ would have the same distribution; (d) Remaining energy to be dissipated per unit surface, $\phi_{\mathrm{S}}=\frac{\Phi_{\mathrm{S}}}{A}$, by the CZM, assuming a transition from CDM to CZM for a given value of damage $D_{\mathrm{T}}$ reached at $x_{0}$.

The length of the bar, $L$, has no effect on the results as it is much longer than the size of the localization zone, so we use $L=1.0 \mathrm{~m}$ for simplicity. We assume a uniform monotonic increasing strain $\varepsilon$, which indicates a monotonic increasing $\tilde{e}=e=\varepsilon$ before strain localization in the 1D bar occurs. The maximum diffuse damage $D_{\text {hom }}$ in equation (41) corresponds to the damage value reached at the peak stress -strain softening onset- in Fig. 4(a), and can be computed by solving $\frac{\mathrm{d} \sigma}{\mathrm{d} \varepsilon}=0$, leading to

$$
\max \left(\varepsilon_{\text {hom }}\right)=0.0238 \text { and } D_{\text {hom }}=0.5093 .
$$

From this value, the system of Eqs. (39-42) completed by Eq. (45) can be solved and the obtained results are presented in Fig. 4(b). This figure illustrates the relation between the traction and the displacement jump of the CZM to be equivalent to the CDM. However this figure is only valid when high damage values arise in the localization zone since the approximated equivalent strain distribution $\tilde{e}(x)(45)$ is only valid for high damage state. For the considered damage model it was found that the approximation is within $1 \%$ error for damage $\geq 0.988$ in the localization zone. The evolution of the stress (41) in terms of $D_{\max }$ is illustrated in Fig. 4(c). Assuming a transition from CDM to CZM when a given value of damage $D_{\mathrm{T}}$ is reached at $x_{0}$, the TSL model can be constructed from the stress $\sigma_{\mathrm{T}}$ reaches at the transition point, see Fig. $4(\mathrm{c})$, and from the remaing energy per unit surface, $\phi_{\mathrm{S}}$, to be dissipated. This latter value can be computed from (32) and its dependance with $D_{\mathrm{T}}$ is illustrated in Fig. 4(d).

The $\sigma-\llbracket u \rrbracket$ and $\phi_{\mathrm{S}}-D_{\mathrm{T}}$ relations are ready to be used in the transition from a continuous CDM to a discontinuous CZM in the simulation of fracture. 


\subsection{Transition from a CDM to a CZM in a $3 D$ setting}

The approach follows the one-dimensional setting developed previously and is applied to three dimensional cases by assuming that the equations of the one dimensional problem are representative of the line perpendicular to the crack front. What remains to be defined is the criterion governing this shift in the expression of the resulting TSL in a 3D setting.

As developed by other researchers [32, 28, 38, 39], the crack nucleation can be governed by a critical transition damage $D_{\mathrm{T}}$. The crack propagation direction is either pre-defined, such as in the intrinsic cohesive model, or evaluated through the damage gradient, such as in xFEM framework or when using remeshing techniques.

In this work, although the cracking path is not pre-defined, the crack propagation needs to follow the element boundaries. If we use the critical damage $D_{\mathrm{T}}$ to trigger crack initiation, the elements may burst apart since the critical damage can be reached on every boundary of a single element. Physically, once a crack form there is unloading in the neighboring elements and a crack cannot be formed on every side of a single element in the static case. Therefore, we assume that the crack nucleation at an interface element is governed by the effective stress

$$
\hat{\sigma}_{\text {eff }}=\left\{\begin{array}{ll}
\sqrt{\hat{\sigma}^{2}+\beta^{-2} \hat{\tau}^{2}} & \text { if } \quad \hat{\sigma} \geqslant 0 \\
\frac{1}{\beta} \ll|\hat{\tau}|-\eta|\hat{\sigma}| \gg & \text { if } \quad \hat{\sigma}<0
\end{array},\right.
$$

where $\hat{\sigma}=\boldsymbol{n} \cdot \hat{\boldsymbol{\sigma}} \cdot \boldsymbol{n}$ and $\hat{\tau}=\sqrt{\boldsymbol{n} \cdot \hat{\boldsymbol{\sigma}} \cdot \boldsymbol{n} \cdot \hat{\boldsymbol{\sigma}}-\hat{\sigma}^{2}}$ are respectively the normal and tangent components of the effective surface traction $\frac{\bar{t}}{1-D}$ at the interface. These components are computed from the effective stress tensor (11), the parameter $\beta=\frac{K_{I I C}}{K_{I C}}$ assigns different weights to the mode I and mode II crack opening, $\eta$ is the friction coefficient of the material, and the operator $\ll \bullet \gg$ refers to the positive value, i.e. zero in case the argument is negative. Equation (47) implicitly accounts for the different behaviors of the material under tension and compression.

Once, the effective cohesive stress reaches the critical effective strength, i.e. $\hat{\sigma}_{\text {eff }} \geqslant \sigma_{\mathrm{C}}$ the fracture framework substitutes to the damage one, and the TSL constructed in Section 2.2.4 is used to evaluate the traction $\overline{\boldsymbol{t}}$ between the crack lips. For simplicity we consider a linear TSL [20, 47] as illustrated in Figure 1(b) to evaluate the traction $\overline{\boldsymbol{t}}$ between the crack lips. As discussed in [48] the shape of the cohesive law is not the main governing factor of the fracture response, so for simplicity we select a linear TSL. This is particularly justified in this work as the transition from CDM to CZM occurs at high values of the damage.

However, in this paper we do not have to account for the triaxiality effect in the cohesive model. Indeed the cohesive zone being activated for high damage values only, the apparent Poisson effect is almost nonexistent at the transition onset. As the effect of stress-triaxiality in a cohesive model is directly related to the Poisson effect [49], there is no need to introduce the stress triaxiality in the TSL. However in a future work we will extend our work to other damage models as the Gurson one for ductile materials [50]. In that case it might be necessary to introduce the crack for lower values of the damage. Also, the Gurson model will not reduce the Poisson effect. For these reasons the cohesive model will have to consider the stress triaxiality effect by accounting for the in-plane stretch as proposed in $[49,51]$.

The effective surfaces separation $\Delta^{*}$ is evaluated from the surface opening vector $\Delta^{*}$ by

$$
\Delta^{*}=\sqrt{\ll \Delta_{\mathrm{n}} \gg^{2}+\beta^{2} \Delta_{\mathrm{t}}^{2}},
$$

where $\Delta_{\mathrm{n}}=\llbracket \boldsymbol{u} \rrbracket \cdot \boldsymbol{n}$ is the separation along the interface element normal $\boldsymbol{n}$, and where $\Delta_{\mathrm{t}}=\llbracket \boldsymbol{u} \rrbracket-\Delta_{\mathrm{n}} \boldsymbol{n}$ is the separation along the interface element tangent $\boldsymbol{t}=\frac{\llbracket \boldsymbol{u} \rrbracket-\Delta_{\mathrm{n}} \boldsymbol{n}}{\left\|\llbracket \boldsymbol{u} \rrbracket-\Delta_{\mathrm{n}} \boldsymbol{n}\right\|}$.

The linear cohesive law, shown in Fig. 1(b), includes an irreversible softening part during the crack opening and a reversible part if a crack unloading occurs. In the latter, the reversible path consists in a line segment which connects the origin with the point corresponding to the maximum opening reached $\left(\Delta_{\max }^{*}\right.$, $\left.\bar{t}_{\max }\right)$. The effective cohesive traction can then be computed from

$$
\bar{t}= \begin{cases}\left(1-D_{\mathrm{T}}\right) \sigma_{\mathrm{C}}\left(1-\frac{\Delta^{*}}{\Delta_{\mathrm{C}}}\right) & \text { for } \dot{\Delta}^{*} \geqslant 0, \text { and } \Delta^{*}=\Delta_{\max }, \\ \bar{t}_{\max } \frac{\Delta^{*}}{\Delta_{\max }} & \text { for } \dot{\Delta}^{*}<0, \text { or } \Delta^{*}<\Delta_{\max },\end{cases}
$$


where $D_{\mathrm{T}}$ is the damage at crack initiation. However, for small $\Delta_{\max }$ a reversible unloading is not allowed in order to avoid instabilities in the explicit time integration.

Finally the cohesive traction vector $\overline{\boldsymbol{t}}$ can be evaluated as a function of the effective cohesive traction $\bar{t}$, following

$$
\overline{\boldsymbol{t}}= \begin{cases}\bar{t}\left(\frac{\Delta_{\mathrm{n}}^{*}}{\Delta^{*}} \boldsymbol{n}+\beta \frac{\left|\Delta_{\mathrm{t}}^{*}\right|}{\Delta^{*}} \boldsymbol{t}\right) & \text { for } \hat{\sigma} \geqslant 0, \\ \bar{t} \beta \frac{\left|\Delta_{t}^{*}\right|}{\Delta^{*}} \boldsymbol{t} & \text { for } \hat{\sigma}<0 .\end{cases}
$$

Note that at fracture initiation as the effective opening is zero, the ratio $\frac{\Delta_{n}^{*}}{\Delta^{*}}$ and the ratio $\frac{\left|\Delta_{\mathrm{t}}^{*}\right|}{\Delta^{*}}$ are respectively initialized to $\frac{\hat{\sigma}_{0}}{\left.(1-D)_{\mathrm{T}}\right) \sigma_{\mathrm{C}}}$ and $\frac{\hat{\tau}_{0}}{\beta\left(1-D_{\mathrm{T}}\right) \sigma_{\mathrm{C}}}$, with $\hat{\sigma}_{0}$ the normal part of the surface traction at fracture initiation and with $\hat{\tau}_{0}$ the tangential part of the surface traction at fracture initiation, see Eq. (47).

Finally the transition from the damage model to a crack is combined with a threshold in the damage evolution of the CDM. Indeed, physically, the material should be elastically unloaded on the crack lips during the crack opening. However in the strain-softening stage of the CDM, during the crack opening the material could be unloaded by increasing the damage or elastically. To force the elastic unloading in that case the damage evolution is limited to the transition damage value $D_{\mathrm{T}}$.

\section{Hybrid non-local implicit Discontinuous Galerkin/extrinsic cohesive law framework}

Once the CDM-CZM transition theoretical background is defined, one remaining problem is its implementation in an efficient parallel framework. In this section we develop an energetically rigorous and computationally efficient way to introduce a crack in the non-local implicit CDM resolution by combining the extrinsic cohesive law with a discontinuous Galerkin (DG) approach. The DG method is developed to solve the non-local CDM weak formulation, following classical derivations of DG methods for (non-linear) elliptic equations, see $[52,53,54,55,56]$ among many others, while accounting for the existence of cracked surfaces within the continuum as suggested in $[22,23,24,25]$. In this hybrid non-local DG/ECL method, interface elements are therefore inserted between bulk elements at the beginning of the simulation and continuity before the transition to the CZM is ensured by having recourse to the consistent DG interface terms. The extrinsic cohesive law developed in Section 2.3 can thus be integrated on the already existing interface elements without requiring mesh topology changes. The framework allows a high scalability to be reached for a parallel implementation.

Let $\Omega \subset \mathbb{R}^{3}$ be a body subjected to the force per unit mass $\boldsymbol{b}$ and with its boundary surface $\Gamma$ including two parts: the Dirichlet boundary denoted by $\Gamma_{\mathrm{D}}$, on which the displacement $\boldsymbol{u}$ is prescribed by $\overline{\boldsymbol{u}}$, and the Neumann boundary denoted by $\Gamma_{\mathrm{N}}$, on which the traction is prescribed by $\overline{\boldsymbol{t}}$. One always has $\Gamma_{=} \Gamma_{\mathrm{D}} \cup \Gamma_{\mathrm{N}}$ and $\Gamma_{\mathrm{D}} \cap \Gamma_{\mathrm{N}}=\emptyset$. Assuming small displacements $\boldsymbol{u}$, the continuum governing equations read

$$
\begin{aligned}
\rho \ddot{\boldsymbol{u}} & =\boldsymbol{\nabla} \cdot \boldsymbol{\sigma}^{T}+\rho \boldsymbol{b} & & \text { in } \Omega, \\
e & =\tilde{e}-\boldsymbol{\nabla} \cdot(c \boldsymbol{\nabla} \tilde{e}) & & \text { in } \Omega,
\end{aligned}
$$

where $\rho$ is the density, and where the second set of equations results from the implicit non-local Eq. (16) presented in Section 2.1.2.

These equations are completed by the following spacial boundary conditions

$$
\begin{aligned}
& \boldsymbol{u}=\overline{\boldsymbol{u}} \text { on } \Gamma_{\mathrm{D}}, \\
& \boldsymbol{\sigma} \cdot \boldsymbol{n}=\overline{\boldsymbol{t}} \quad \text { on } \Gamma_{\mathrm{N}} \text {, and } \\
& (c \boldsymbol{\nabla} \tilde{e}) \cdot \boldsymbol{n}=0 \quad \text { on } \Gamma \text {. }
\end{aligned}
$$

On the uncracked part of the body the exact solution $\left(\boldsymbol{u}^{\text {exact }}, \tilde{e}^{\text {exact }}\right)$ of the strong form (51-55) is continuous, and so its derivative. So considering any uncracked surface in the body $\Gamma_{U} \in \Omega$ implies

$$
\begin{aligned}
& \llbracket \boldsymbol{u}^{\text {exact }} \rrbracket=0 \text { on } \Gamma_{U}, \quad \llbracket \tilde{e}^{\text {exact }} \rrbracket=0 \text { on } \Gamma_{U}, \\
& \llbracket \boldsymbol{\sigma}^{\text {exact }} \rrbracket=0 \text { on } \Gamma_{U}, \quad \text { and } \llbracket c \boldsymbol{\nabla} \tilde{e}^{\text {exact }} \rrbracket=0 \text { on } \Gamma_{U} .
\end{aligned}
$$


In these equations we have considered the jump operator. This jump operator and the average operator are defined on an interface lying in the body, which separates the parts arbitrarily denoted "plus" and "minus", by respectively

$$
\llbracket \bullet \rrbracket=\left[\bullet^{+}-\bullet^{-}\right] \text {and }\langle\bullet\rangle=\frac{1}{2}\left[\bullet^{+}+\bullet^{-}\right] \text {. }
$$

Considering a cracked surface inside the body $\Gamma_{C} \in \Omega$, the strong form (51-57) is completed by the governing equation, in terms of the surface traction $\overline{\boldsymbol{t}}=\boldsymbol{\sigma} \cdot \boldsymbol{n}$, on the crack lips

$$
\begin{aligned}
& \llbracket \overline{\boldsymbol{t}} \rrbracket=\mathbf{0} \quad \text { on } \quad \Gamma_{C}, \\
& \bar{t}=\|\overline{\boldsymbol{t}}\| \leq \bar{t}_{\max } \quad \text { on } \quad \Gamma_{C}, \\
& \left(\bar{t}_{\max }-\bar{t}\right)\left\{\begin{array}{ll}
>0 & \text { if } \dot{\Delta}^{*}<0 \text { or } \Delta^{*}<\Delta_{\max }^{*} \\
=0 & \text { if } \dot{\Delta}^{*}>0 \text { and } \Delta^{*}=\Delta_{\max }^{*}
\end{array} \text { on } \Gamma_{C},\right. \\
& (c \boldsymbol{\nabla} \tilde{e}) \cdot \boldsymbol{n}=0 \quad \text { on } \quad \Gamma_{C},
\end{aligned}
$$

where $\bar{t}$ represents the surface traction amplitude between the crack lips, where $\Delta^{*}$ is the opening of the crack, and where $\bar{t}_{\text {max }}$ is the surface traction amplitude at the maximum crack opening $\Delta_{\text {max }}^{*}$ reached during the fracture process. Equation (62) assumes that once the crack is introduced in the discretization, the crack surfaces act as free boundaries with regards to the non-local implicit equations.

The finite element discretization of the body reads $\Omega=\bigcup_{e} \bar{\Omega}^{e}$, where $\bar{\Omega}^{e}$ is the union of the open element domain $\Omega^{e}$ with its boundary $\Gamma^{e}$. Here the symbol $\Omega$ is used to represent the body and its discretization for simplicity. The weak form of Equations (51-55) is established by approximating the unknows fields on $\Omega$ with a polynomial approximation $(\boldsymbol{u}, \tilde{e})$. Contrarily to a continuous Galerkin approximation, which requires continuous interpolations, the DG approach only requires an element-wise continuous polynomial approximation, i.e. $(\boldsymbol{u}, \tilde{e}) \in C^{0}\left(\Omega^{e}\right)$. Thus the trial functions $\boldsymbol{w}_{u}$ and $w_{\tilde{e}}$, of the displacement and of the non-local fields respectively, are also discontinuous across the element interfaces on the internal boundary of the body $\Gamma_{\mathrm{I}}=\left[\bigcup_{e} \Gamma^{e}\right] \backslash \Gamma$. In the context of the hybrid DG/ECL approach herein extended to the non-local implicit formulation, the internal boundary $\Gamma_{\mathrm{I}}$ includes uncracked surfaces $\Gamma_{\mathrm{IU}}=\Gamma_{\mathrm{I}} \cap \Gamma_{\mathrm{U}}$ on which the strong form (56-57) holds, and cracked surfaces $\Gamma_{\mathrm{IC}}=\Gamma_{\mathrm{I}} \cap \Gamma_{\mathrm{C}}$ on which the strong form (59-62) holds.

The linear momentum balance is enforced in a weighted-average sense by multiplying the strong form (51-52) by suitable test functions, respectively $\boldsymbol{w}_{u}$ and $w_{\tilde{e}}$, and by integrating by parts on each element instead of performing this integration on the uncracked domain as it is usually performed for a continuous Galerkin approximation. Using traditional DG considerations for elliptic equations to treat the uncracked part of the body, see [52] e.g. for details, and defining $\overline{\boldsymbol{t}}^{-}$as the surface traction evaluated on the minus side of the cracked surfaces, lead to the weak form

$$
\begin{array}{r}
\int_{\Omega}\left(\rho \ddot{\boldsymbol{u}} \cdot \boldsymbol{w}_{u}+\boldsymbol{\sigma}: \boldsymbol{\nabla} \boldsymbol{w}_{u}\right) d V+\int_{\Gamma_{\mathrm{IC}}} \llbracket \boldsymbol{w}_{u} \rrbracket \cdot \overline{\boldsymbol{t}}^{-}(\llbracket \boldsymbol{u} \rrbracket) d s+ \\
\int_{\Gamma_{\mathrm{IU}}} \llbracket \boldsymbol{w}_{u} \rrbracket \cdot\langle\boldsymbol{\sigma}\rangle \cdot \boldsymbol{n}^{-} d s=\int_{\Omega} \rho \boldsymbol{b} \cdot \boldsymbol{w}_{u} d V+\int_{\Gamma_{\mathrm{N}}} \boldsymbol{w}_{u} \cdot \overline{\boldsymbol{t}} d s \\
\int_{\Omega}\left(\tilde{e} w_{\tilde{e}}+\nabla w_{\tilde{e}} \cdot c \boldsymbol{\nabla} \tilde{e}\right) d V+\int_{\Gamma_{\mathrm{IU}}} \llbracket w_{\tilde{e}} \rrbracket\langle c \boldsymbol{\nabla} \tilde{e}\rangle \cdot \boldsymbol{n}^{-} d s \\
=\int_{\Omega} e w_{\tilde{e}} d V
\end{array}
$$

where $\boldsymbol{n}^{-}$is the outward unit surface normal of the "minus" element.

In the formulation (63-64), neither the inter-element field continuity is enforced across the uncracked surfaces $\Gamma_{\mathrm{IU}}$, nor the stability of the method. The compatibility equations $\boldsymbol{u}^{-}-\boldsymbol{u}^{+}=0$ and $\tilde{e}^{-}-\tilde{e}^{+}=0$ are enforced on $\Gamma_{\text {IU }}$ using the so-called symmetrization terms and sufficiently large quadratic stabilization terms. With the addition of the quadratic terms, the fields jumps are stabilized in the numerical solution, while the symmetrization terms lead to an optimal convergence rate with respect to the mesh size. The final 
weak formulation of the problem is stated as finding $(\boldsymbol{u}, \tilde{e})$ such that

$$
\begin{aligned}
& \int_{\Omega}\left(\rho \ddot{\boldsymbol{u}} \cdot \boldsymbol{w}_{u}+\boldsymbol{\sigma}: \nabla \boldsymbol{w}_{u}\right) d V+\int_{\Gamma_{\mathrm{IC}}} \llbracket \boldsymbol{w}_{u} \rrbracket \cdot \overline{\boldsymbol{t}}^{-}(\llbracket \boldsymbol{u} \rrbracket) d s+ \\
& \int_{\Gamma_{\mathrm{IU}}} \llbracket \boldsymbol{w}_{u} \rrbracket \cdot\langle\boldsymbol{\sigma}\rangle \cdot \boldsymbol{n}^{-} d s+\int_{\Gamma_{\mathrm{IU}}} \llbracket \boldsymbol{w}_{u} \rrbracket \otimes \boldsymbol{n}^{-}:\left\langle\frac{\beta_{s}}{h_{s}} \mathbb{C}\right\rangle: \llbracket \boldsymbol{u} \rrbracket \otimes \boldsymbol{n}^{-} d s+ \\
& \int_{\Gamma_{\mathrm{IU}}} \llbracket \boldsymbol{u} \rrbracket \cdot\left\langle\mathbb{C}: \nabla_{0} \boldsymbol{w}_{u}\right\rangle \cdot \boldsymbol{n}^{-} d s=\int_{\Omega} \rho \boldsymbol{b} \cdot \boldsymbol{w}_{u} d V+\int_{\Gamma_{\mathrm{N}}} \boldsymbol{w}_{u} \cdot \overline{\boldsymbol{t}} d s, \\
& \int_{\Omega}\left[\tilde{e} w_{\tilde{e}}+\nabla w_{\tilde{e}} \cdot(c \nabla \tilde{e})\right] d V+\int_{\Gamma_{\mathrm{IU}}} \llbracket w_{\tilde{e}} \rrbracket\langle c \nabla \tilde{e}\rangle \cdot \boldsymbol{n}^{-} d s+ \\
& \int_{\Gamma_{\mathrm{IU}}} \llbracket w_{\tilde{e}} \rrbracket \boldsymbol{n}^{-} \cdot\left\langle\frac{\beta_{s}}{h_{s}} c\right\rangle \cdot \boldsymbol{n}^{-} \llbracket \tilde{e} \rrbracket d s+\int_{\Gamma_{\mathrm{IU}}} \llbracket \tilde{e} \rrbracket\left\langle c \nabla w_{\tilde{e}}\right\rangle \cdot \boldsymbol{n}^{-} d s=\int_{\Omega} e w_{\tilde{e}} d V
\end{aligned}
$$

$\forall\left(\boldsymbol{w}_{u}, w_{\tilde{e}}\right)$ kinematically admissible. In this set of equations, $h_{s}$ is the mesh size and $\beta_{s}$ is the penalty parameter for stabilization. In Eq. (65) the elastic operator $\mathbb{C}$ has been used instead of the algorithmic operator $\frac{\partial \boldsymbol{\sigma}}{\partial \boldsymbol{\varepsilon}}$. Indeed, in order to ensure the stability of the method, the quadratic terms cannot vanish, which would be the case with the latter choice during the strain softening.

In the weak form (65-66), the stress tensor $\boldsymbol{\sigma}$ is obtained from the elastic damage model reported in Section 2.1.2, with the damage evolution (14). The traction on cracked surfaces follows the governing equation (50) constructed in Section 2.3 from the strength and energy defined in Section 2.2.4.

In this DG/ECL framework, both the DG surface terms $\Gamma_{\text {IU }}$ and the CZM terms $\Gamma_{\text {IC }}$ are integrated using interface elements. Considering initially a Gauss point on such an uncracked interface, the onset of fracture is detected following Section 2.3, and when this criterion is met, a crack nucleates. This Gauss point is thus no longer associated to the uncracked surface $\Gamma_{\mathrm{IU}}$, but to the cracked part $\Gamma_{\mathrm{IC}}$. Practically, only the governing law (from DG to TSL) is substituted at crack initiation, which prevents mesh or topology modifications and makes the implementation computationally efficient. ${ }^{2}$

\section{Finite element discretization and resolution}

In this section the weak formulation (65-66) of the non-local implicit DG/ECL framework is taken as the basis for the finite element discretization. A staggered resolution scheme is also proposed before discussing the numerical properties of the method.

\footnotetext{
${ }^{2}$ For completeness, in the context of the DG formulation one slight change has to be done in the definition of the effective opening $\Delta^{*}(48)$ evaluated from the surface opening vector $\boldsymbol{\Delta}^{*}$ to account for the jumps $\llbracket \boldsymbol{u}_{0} \rrbracket$ existing in the DG formulation that precedes to the crack insertion. The effective opening (48) is redefined as

$$
\Delta^{*}=\sqrt{\ll \Delta_{\mathrm{n}}^{*} \gg^{2}+\beta^{2} \Delta_{\mathrm{t}}^{* 2}},
$$

where $\Delta_{\mathrm{n}}^{*}$ and $\Delta_{\mathrm{t}}^{*}$ are redefined as

$$
\left\{\begin{array}{l}
\llbracket \boldsymbol{u} \rrbracket^{*}=\llbracket \boldsymbol{u} \rrbracket-\llbracket \boldsymbol{u} \rrbracket_{0} \\
\Delta_{\mathrm{n}}^{*}=\llbracket \boldsymbol{u} \rrbracket^{*} \cdot \boldsymbol{n} \\
\Delta_{\mathrm{t}}^{*}=\llbracket \boldsymbol{u} \rrbracket^{*} \cdot \boldsymbol{t}
\end{array} .\right.
$$

With these changes, the tangential direction is evaluated from $\boldsymbol{t}=\frac{\llbracket \boldsymbol{u} \rrbracket^{*}-\Delta_{n}^{*} \boldsymbol{n}}{\left\|\llbracket \boldsymbol{u} \rrbracket^{*}-\Delta_{n}^{*} \boldsymbol{n}\right\|}$ during opening.
} 


\subsection{Finite element formulation}

The displacement mapping $\boldsymbol{u}$ and the effective strain $\tilde{e}$ are approximated by the same interpolation in each element $\Omega^{e}$, simultaneously as their respective arbitrary trial functions, following,

$$
\begin{array}{rlrl}
\boldsymbol{u}(\boldsymbol{X}) & =\sum_{a=1}^{N} N^{a}(\boldsymbol{X}) \boldsymbol{u}^{a}, & & \boldsymbol{w}_{u}(\boldsymbol{X})=\sum_{a=1}^{N} N^{a}(\boldsymbol{X}) \delta \boldsymbol{u}^{a}, \text { and } \\
\tilde{e}(\boldsymbol{X})=\sum_{a=1}^{N} N^{a}(\boldsymbol{X}) \tilde{e}^{a}, & \boldsymbol{w}_{\tilde{e}}(\boldsymbol{X})=\sum_{a=1}^{N} N^{a}(\boldsymbol{X}) \delta \tilde{e}^{a},
\end{array}
$$

where $N_{a}$ is the traditional shape function corresponding to the node $a \in\{1, \ldots, N\}$, with $N$ being the number of nodes. We define $\mathbf{q}^{a T}=\left[\boldsymbol{u}^{a T} \tilde{e}^{a}\right]$ as the nodal vector of unknowns and $\mathbf{q}^{T}=\left[\mathbf{u}^{T} \tilde{\mathbf{e}}\right]$ as the vector regrouping the degrees of freedom of the whole mesh. In the presented framework the unknown fields, the arbitrary trial functions, and the shape functions are discontinuous across the elements interfaces.

Applying the discretization process, the finite-element forces can be computed, see Appendix B for details, and the weak form (65-66) reduces to a set of ordinary differential equations to be integrated in the time interval $T$, which are expressed in the matrix form as

$$
\begin{array}{cc}
\mathbf{M} \ddot{\mathbf{u}}+\mathbf{f}_{\boldsymbol{u} \text { int }}(\mathbf{q})+\mathbf{f}_{\boldsymbol{u} \mathbf{I}}(\mathbf{q})=\mathbf{f}_{\boldsymbol{u} \text { ext }} & \forall t \in T, \\
\mathbf{f}_{\tilde{e} \operatorname{int}}(\mathbf{q})-\mathbf{f}_{e \operatorname{int}}(\mathbf{q})+\mathbf{f}_{\tilde{e} \mathrm{I}}(\mathbf{q})=0 & \forall t \in T,
\end{array}
$$

where $\mathbf{M}$ is the discretized mass matrix, and where the expressions of $\mathbf{f}_{\boldsymbol{u} \text { int }}, \mathbf{f}_{\boldsymbol{u}}, \mathbf{f}_{\boldsymbol{u} \text { ext }}$, respectively the internal, interface and external force vectors related to the displacement field and $\mathbf{f}_{\tilde{e} \text { int }}, \mathbf{f}_{e \text { int }}, \mathbf{f}_{\tilde{e} \mathrm{I}}$, respectively the non-local internal, local internal and non-local interface force vectors related to the non-local effective degrees of freedom, are reported in Appendix B. The set of Eqs. (71) is completed by the initial conditions $\boldsymbol{u}^{a}(t=0)=0$ and $\dot{\boldsymbol{u}}^{a}(t=0)=\mathbf{v}_{0}^{a}$, where $\mathbf{v}_{0}^{a}$ are the initial nodal velocities. The time interval of interest $T$ is discretized into time steps and the integration is accomplished through an incremental solution procedure in each time interval $\left[t^{n}, t^{n+1}\right]$.

At a given time $t^{n+1}$, the linearization of the system of Eqs. (71-72) around the field $\mathbf{q}^{i}$ obtained at iteration $i$ reads

$$
\begin{aligned}
& \mathbf{M} \delta \ddot{\mathbf{u}}+\underbrace{\frac{\partial \mathbf{f}_{\boldsymbol{u}} \text { int }}{\partial \mathbf{u}}}_{\mathbf{K}_{\boldsymbol{u} u \text { int }}} \delta \mathbf{u}+\underbrace{\frac{\partial \mathbf{f}_{\boldsymbol{u}} \text { int }}{\partial \tilde{\mathbf{e}}}}_{\mathbf{K}_{\boldsymbol{u} \tilde{e} \text { int }}} \delta \tilde{\mathbf{e}}+\underbrace{\frac{\partial \mathbf{f}_{\boldsymbol{u}} \mathrm{I}}{\partial \mathbf{u}}}_{\mathbf{K}_{\boldsymbol{u} u \mathrm{I}}} \delta \mathbf{u}+\underbrace{\frac{\partial \mathbf{f}_{\boldsymbol{u}} \mathrm{I}}{\partial \tilde{\mathbf{e}}}}_{\mathbf{K}_{\boldsymbol{u} \tilde{e} \mathrm{I}}} \delta \tilde{\mathbf{e}}= \\
& \mathbf{f}_{\boldsymbol{u} \text { ext }}-\mathbf{f}_{\boldsymbol{u} \text { int }}\left(\mathbf{q}^{i}\right)-\mathbf{f}_{\boldsymbol{u} \mathrm{I}}\left(\mathbf{q}^{i}\right) \text {, and } \\
& (\underbrace{\frac{\partial \mathbf{f}_{\tilde{e} \text { int }}}{\partial \mathbf{u}}}_{\mathbf{K}_{\tilde{e} \boldsymbol{u} \text { int }}}-\underbrace{\frac{\partial \mathbf{f}_{e \text { int }}}{\partial \mathbf{u}}}_{\mathbf{K}_{e \mathbf{u} \text { int }}}) \delta \mathbf{u}+(\underbrace{\frac{\partial \mathbf{f}_{\tilde{e} \text { int }} \partial \tilde{\mathbf{e}}}{\partial \mathbf{f}_{\tilde{e}}}}_{\mathbf{K}_{\tilde{e} \tilde{e} \text { int }}}-\underbrace{\frac{\partial \mathbf{f}_{e i n t}}{\partial \tilde{\mathbf{e}}}}_{\mathbf{K}_{e \tilde{e} \text { int }}}) \delta \tilde{\mathbf{e}}+ \\
& \underbrace{\frac{\partial \mathbf{f}_{\tilde{e} \mathrm{I}}}{\partial \boldsymbol{u}}}_{\mathbf{K}_{\tilde{e} \boldsymbol{u} \mathrm{I}}} \delta \boldsymbol{u}+\underbrace{\frac{\partial \mathbf{f}_{\tilde{e} \mathrm{I}}}{\partial \tilde{e}}}_{\mathbf{K}_{\tilde{e} \tilde{e} \mathrm{I}}} \delta \tilde{e}=\mathbf{f}_{e \operatorname{int}}\left(\mathbf{q}^{i}\right)+\mathbf{f}_{\tilde{e} \mathrm{I}}\left(\mathbf{q}^{i}\right)-\mathbf{f}_{\tilde{e} \operatorname{int}}\left(\mathbf{q}^{i}\right),
\end{aligned}
$$

where the $\mathbf{K}_{\ldots}$.. are the different contributions to the stiffness matrix whose expressions are reported in Appendix B. The linearized system (73-74) can be rewritten under the form

$$
\begin{array}{r}
{\left[\begin{array}{ll}
\mathbf{M} & \mathbf{0} \\
\mathbf{0} & \mathbf{0}
\end{array}\right]\left[\begin{array}{l}
\delta \ddot{\boldsymbol{u}} \\
\mathbf{0}
\end{array}\right]+\left[\begin{array}{ll}
\mathbf{K}_{\boldsymbol{u} \boldsymbol{u}} & \mathbf{K}_{\boldsymbol{u} \tilde{e}} \\
\mathbf{K}_{\tilde{e} \boldsymbol{u}} & \mathbf{K}_{\tilde{e} \tilde{e}}
\end{array}\right]\left[\begin{array}{l}
\delta \boldsymbol{u} \\
\delta \tilde{e}
\end{array}\right]=} \\
{\left[\begin{array}{l}
\mathbf{f}_{\boldsymbol{u} \operatorname{ext}}-\mathbf{f}_{\boldsymbol{u} \text { int }}\left(\mathbf{q}^{i}\right)-\mathbf{f}_{\boldsymbol{u} \mathrm{I}}\left(\mathbf{q}^{i}\right) \\
\mathbf{f}_{e \operatorname{int}}\left(\mathbf{q}^{i}\right)+\mathbf{f}_{\tilde{e} \mathrm{I}}\left(\mathbf{q}^{i}\right)-\mathbf{f}_{\tilde{e} \operatorname{int}}\left(\mathbf{q}^{i}\right)
\end{array}\right]}
\end{array}
$$


where $\mathbf{K}_{\boldsymbol{u} \boldsymbol{u}}=\mathbf{K}_{\boldsymbol{u} \boldsymbol{u} \text { int }}+\mathbf{K}_{\boldsymbol{u} \boldsymbol{u} \mathrm{I}}, \mathbf{K}_{\boldsymbol{u} \tilde{e}}=\mathbf{K}_{\boldsymbol{u} \tilde{e} \text { int }}+\mathbf{K}_{\boldsymbol{u} \tilde{e} \mathrm{I}}, \mathbf{K}_{\tilde{e} \boldsymbol{u}}=\mathbf{K}_{\tilde{e} \boldsymbol{u} \text { int }}-\mathbf{K}_{e \boldsymbol{u} \text { int }}+\mathbf{K}_{\tilde{e} \boldsymbol{u} \mathrm{I}}$, and $\mathbf{K}_{\tilde{e} \tilde{e}}=\mathbf{K}_{\tilde{e} \tilde{e} \text { int }}-$ $\mathbf{K}_{e \tilde{e} i n t}+\mathbf{K}_{\tilde{e} \tilde{e} \mathrm{I}}, \mathbf{f}_{\text {int }}$ is the discretized internal forces vector, $\mathbf{f}_{\mathrm{I}}$ is the discretized interface forces vector, and where $\mathbf{f}_{\text {ext }}$ is the discretized external forces vector.

On the one hand, assuming no inertial effects, this system can be solved in a fully coupled way using a tradition static non-linear solver. On the other hand, the dynamics Eq. (71) can be integrated using an explicit time integration. The scheme considered herein is the second-order accurate Hulbert-Chung time integration [57], which exhibits numerical dissipation. Knowing the solution at time $t^{n}$, the solution at time $t^{n+1}$ can be obtained by the expressions

$$
\begin{aligned}
\ddot{\boldsymbol{u}}_{b}^{n+1} & =\frac{1}{1-\alpha_{M}} \sum_{b=1}^{N} \mathbf{M}_{a b}^{-1}\left[\mathbf{f}_{\mathrm{ext} a}^{n}-\mathbf{f}_{\mathrm{int} a}^{n}-\mathbf{f}_{\mathrm{I} a}^{n}\right]-\frac{\alpha_{M}}{1-\alpha_{M}} \ddot{\boldsymbol{u}}_{b}^{n}, \\
\dot{\boldsymbol{u}}_{b}^{n+1} & =\dot{\boldsymbol{u}}_{b}^{n}+\Delta t\left[1-\gamma_{M}\right] \ddot{\boldsymbol{u}}_{b}^{n}+\Delta t \gamma_{M} \ddot{\boldsymbol{u}}_{b}^{n+1}, \text { and } \\
\boldsymbol{u}_{b}^{n+1} & =\boldsymbol{u}_{b}^{n}+\Delta t \dot{\boldsymbol{u}}_{b}^{n}+\Delta t^{2}\left[\frac{1}{2}-\beta_{M}\right] \ddot{\boldsymbol{u}}_{b}^{n}+\Delta t^{2} \beta_{M} \ddot{\boldsymbol{u}}_{b}^{n+1},
\end{aligned}
$$

where the parameters $\alpha_{M}, \beta_{M}$ and $\gamma_{M}$ are defined according to the desired numerical dissipation ${ }^{3}$, see [57] for more details. In this case, the remaining non-local equation of the system (75) becomes

$$
\mathbf{K}_{\tilde{e} \tilde{e}} \delta \tilde{e}=\mathbf{f}_{e \text { int }}\left(\mathbf{q}^{i}\right)+\mathbf{f}_{\tilde{e} \mathrm{I}}\left(\mathbf{q}^{i}\right)-\mathbf{f}_{\tilde{e} \text { int }}\left(\mathbf{q}^{i}\right) .
$$

This equation does not have to be solved at every explicit time-step as it is unconditionally stable and as it would induce an over-cost. Practically this last system is resolved every 100-1000 explicit steps. Such a staggered techniques was previously proposed in [29].

As extensive computations will be required due to the need for fine meshes, the framework is implemented in parallel in Gmsh [58] following the scalable face-based ghost implementation presented in [25, 27] for classical mechanics and adapted in a straightforward way to the non-local implicit scheme.

\subsection{Numerical properties}

The numerical properties, related to the spatial discretization, of the Interior Penalty (IP) formulation (65-66), before crack onset, i.e. $\Gamma_{\mathrm{I}}=\Gamma_{\mathrm{IU}}$, can be derived by following exactly the path set for other elliptic problems $[52,59]$. However although the second set of equations (66) is linear, the first set of equations (65) is not and the properties rigorously hold in the linearized form only. These properties can be summarized as (i) the method is consistent, i.e. the exact solution of the problem satisfies the equations (65-66), (ii) the method is stable if the stability parameter $\beta_{s}$ is larger than a constant that depends on the finite element polynomial order only, (iii) the convergence with the mesh size $h_{s}$ in the energy norm is at order $k-1$ for a polynomial approximation of order $k$, and (iv) the convergence with the mesh size $h_{s}$ in the $\mathrm{L}^{2}$-norm is at order $k+1$ for a polynomial approximation of order $k$. Finally, the explicit time-integration (76-78) is conditionally stable and the time step should satisfy the Courant-Friedrichs-Lewy condition $\Delta t=t^{n+1}-t^{n}<\Delta t_{\text {crit }}$, where $\Delta t_{\text {crit }}$ is the critical time-step, which is divided by $\sqrt{\beta_{s}}$ compared to the usual critical time step to account for the DG space-discretization, i.e. $\Delta t<\frac{h_{s} \Omega_{S}}{2 \sqrt{\beta_{s} c_{s}}}$ where $\Omega_{S} \leq 2$ is the stability non-dimensional frequency of the time-integration algorithm [57] and $c_{s}$ is the sound speed, see [54].

Concerning the properties related to the crack propagation, as the method corresponds to an extrinsic CZM, for which the crack path and the dissipated energy converge with the mesh size for unstructured meshes [40, 41], the hybrid scheme also inherits these properties as it has recently been orally discussed in [60]. We will demonstrate in the numerical application that the results are insensitive with the mesh size. Note that it has been pointed out that the reversible unloading curve of the CZM cannot be too steep not to affect the method stability [25].

\footnotetext{
${ }^{3}$ To be stable, a time integration algorithm must either conserve the system energy or dissipate part of it during a time--step computation. Depending on the selected parameters, the Hulbert-Chung algorithm either conserves the energy or numerically dissipates its higher frequencies contributions. In the latter, this so-called numerical dissipation stabilizes the simulation as oscillations related to the purely numerical modes are damped.
} 


\section{Numerical applications}

In this section the effect of the transition criterion is first investigated on the Compact-Tension specimen (CTS). Then the results for the Double-Notched specimen (DNS) are compared for different mesh sizes.

\subsection{Compact-Tension specimen}

The geometry of the specimen is given in Fig. 5(a), and the dimensions are $W=50 \mathrm{~mm}, a_{\mathrm{n}}=10 \mathrm{~mm}$ and thickness $t=3.8 \mathrm{~mm}$. The reference experimental results come from the benchmark work of Geers [6], in which the CTS test was carried out on short glass-fiber-reinforced polypropylene.

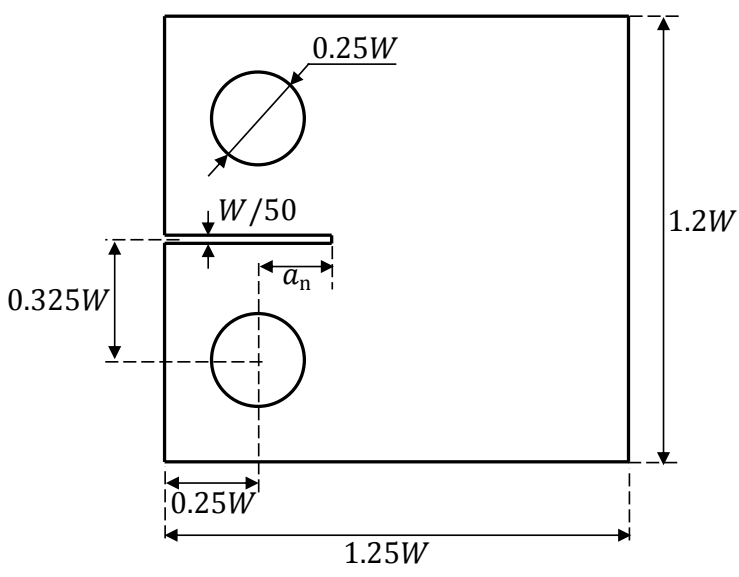

(a) Geometry

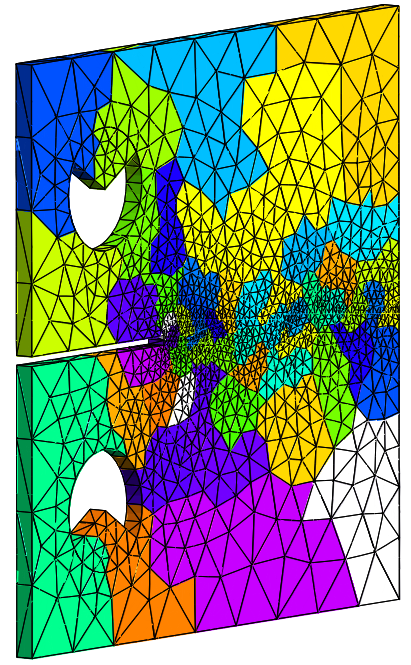

(b) Mesh

Figure 5: The Compact-Tension specimen. (a) Geometry with $W=50 \mathrm{~mm}, a_{\mathrm{n}}=10 \mathrm{~mm}$ and thickness $t=3.8 \mathrm{~mm}$. (b) Partitioned mesh with the different partitions in different colors.

Table 1: Material properties and models parameters [6].

\begin{tabular}{lll}
\hline Elastic material properties, Eq. (11) & & \\
Young's modulus & $E$ & $3.2[\mathrm{GPa}]$ \\
Poisson's ratio & $\nu$ & 0.28 \\
\hline Parameters of damage model, Eq. (14) & $\kappa_{\mathrm{i}}$ & 0.011 \\
& $\kappa_{\mathrm{C}}$ & 0.5 \\
& $\alpha$ & 5.0 \\
& $\beta$ & 0.75 \\
\hline Parameters of characteristic length model, Eq. (20) & & \\
& $c_{0}$ & $2.0\left[\mathrm{~mm}^{2}\right]$ \\
& $e_{\mathrm{C}}$ & 0.15 \\
& $n_{\mathrm{C}}$ & 1.0 \\
\hline
\end{tabular}

The material follows an isotropic linear elastic constitutive law enhanced by a non-local damage model. Its properties and the parameters of the applied model are summarized in Table 1 [6]. The 3D finite element 
Table 2: CDM-CZM transition parameters [6].

\begin{tabular}{lll}
\hline First set & & \\
Effective critical stress & $\sigma_{\mathrm{C}}$ & $1767[\mathrm{MPa}]$ \\
1D-equivalent damage & $D_{\mathrm{T}}$ & 0.99 \\
Remaining energy & $\phi_{\mathrm{S}}$ & $8.79\left[\mathrm{~kJ} / \mathrm{m}^{2}\right]$ \\
Mix-mode ratio & $\beta$ & 0.87 \\
\hline Second set & & \\
Effective critical stress & $\sigma_{\mathrm{C}}$ & $604.2[\mathrm{MPa}]$ \\
1D-equivalent damage & $D_{\mathrm{T}}$ & 0.95 \\
Remaining energy & $\phi_{\mathrm{S}}$ & $14.4\left[\mathrm{~kJ} / \mathrm{m}^{2}\right]$ \\
Mix-mode ratio & $\beta$ & 0.87 \\
\hline Third set & & \\
Effective critical stress & $\sigma_{\mathrm{C}}$ & $239.9[\mathrm{MPa}]$ \\
1D-equivalent damage & $D_{\mathrm{T}}$ & 0.85 \\
Remaining energy & $\phi_{\mathrm{S}}$ & $17.66\left[\mathrm{~kJ} / \mathrm{m}^{2}\right]$ \\
Mix-mode ratio & $\beta$ & 0.87 \\
\hline
\end{tabular}

model of half of the specimen ${ }^{4}$ is meshed with 6320 10-node tetrahedra with quadratic interpolations for the displacements and for the non-local field. A finer discretization is always applied in the band where the crack is expected to initiate and propagate, while a coarse mesh is used far away from this damage process zone. The loading pins are modeled with elastic quarters of cylinder, with a high stiffness (Young modulus $E=30 G P a)$ compared to the specimen material, to avoid tensile forces transmission between the specimen and the pins. It would be more rigorous to use springs with rigid compression stiffness and zero tensile stiffness to model the loading pins as proposed in [6]. Therefore, an error might be introduced due to the loading model. The mesh is partitioned on 48 processors. Fig. 5(b) illustrates the FE model used in the simulations. The displacements of the axes of the cylinders are controlled in the vertical direction -in other directions the displacements of these axes are constrained to zero- and the rotation of the specimen around the loading axes is allowed.

The transition from the CDM to the CZM has now to be fully characterized. This transition is defined by a value of the effective critical stress $\sigma_{\mathrm{C}}$, following Section 2.3. In a $1 \mathrm{D}$ setting, this corresponds to applying the shift at a damage value $D_{\mathrm{T}}$ obtained accordingly to Fig. 4(c). The remaining energy per unit crack surface $\phi_{\mathrm{S}}$ to be dissipated by the CZM is obtained following Fig. 4(d). Three sets of parameters are successively studied and are reported in Table $2^{5}$. Remember that these results have been obtained assuming the transition occurs at a high damage value. The first set corresponds to a transition at the higher damage and satisfies the assumption required for the approximation carried out in Section 2.2.4, leading to less than $1 \%$ error. Two other transition parameter sets correspond to lower values of the transition damage and thus to a higher error.

The evolution of the loading force (at the pins and for the full specimen) in terms of the relative displacement between the two loading pins is reported in Fig. 6(a) for the three different set of transition parameters. In this figure the experimental results $[6]^{6}$ obtained and the result obtained for a pure CDM simulation without crack insertion are also reported for comparison purpose. The numerical solution corresponding to the first set of transition parameters, i.e. for $D_{\mathrm{T}}=0.99$, are in good agreements with the experimental results during the loading and softening phase. The only difference is the peak loading which is slightly lower. As the deviation with the experimental results appears before the crack initiation, the

\footnotetext{
${ }^{4}$ For symmetry reasons only half of the thickness is considered

${ }^{5}$ As usually done for cohesive model a scatter of $5 \%$ in the critical stress is consider to avoid fracturing all the Gauss points at the same step.

${ }^{6}$ The experimental curves reported in [6] do not pass by the origin so they have been translated in this work
} 


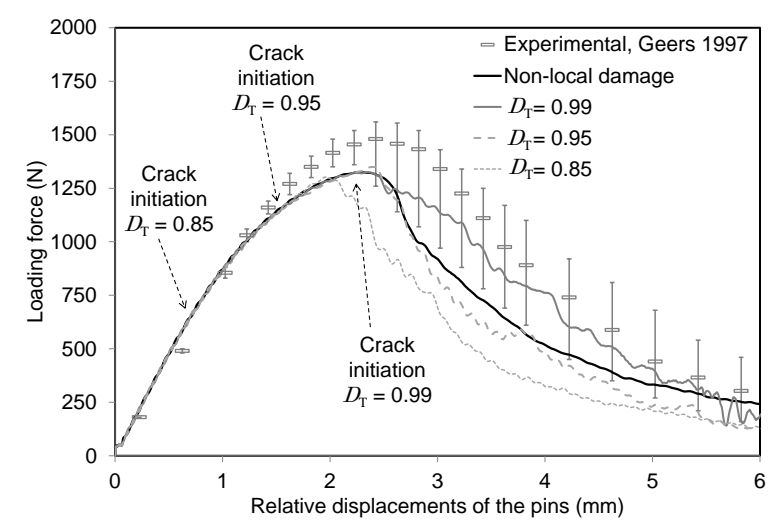

(a) Effect of transition parameters

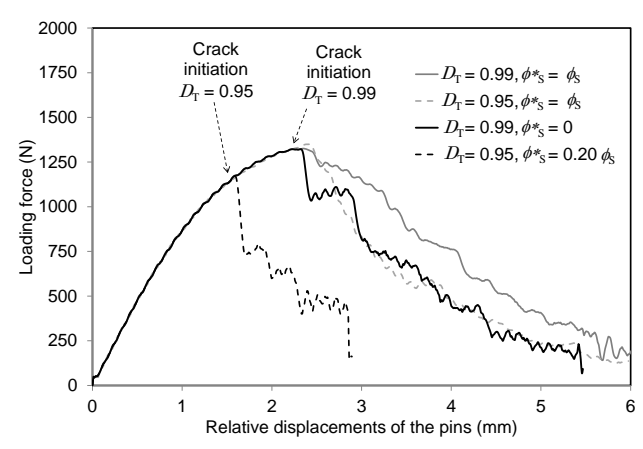

(b) Effect of residual CZM energy

Figure 6: Comparison of the force vs. displacement curves obtained for the Compact-Tension specimen (a) for different sets of transition parameters and (b) for different residual energy in the CZM ( $\phi_{\mathrm{S}}^{*}$ the remaining energy in the CZM actually applied is a part of the consistent value $\left.\phi_{\mathrm{S}}\right)$.

difference is due to the model (loading pins) and not to the CDM-CZM transition. This demonstrates that the transition from CDM to CZM is achieved without loss or jump in the energy, which ensures the accuracy of the method. For the second set of parameters, i.e. for $D_{\mathrm{T}}=0.95$, the crack is initiated before the structural strain softening onset, but because of the introduction of a higher energy $\phi_{S}$ in the CZM, the force keeps increasing and overall dissipates a comparable amout of energy. Finally for the third set of parameters, i.e. $D_{\mathrm{T}}=0.85$, the crack initiates well before the structrual strain softening onset but the load keeps increasing although the peak value is lower than for the other results. This is explained by the error introduced in the approximation carried out in Section 2.2.4 to determine the parameters of the CZM, but also because the energy is extracted from a 1D model which does not consider the triaxiality, leading to a lower dissipation energy [49] for lower damage transition values. As a general observation, introducing the crack earlier leads to a lower force during the strain softening stage although the difference becomes smaller at the end of the simulation, the three curves converging for a relative displacement of $6 \mathrm{~mm}$. Note that the simulation without crack introduction leads to a curve lower than for a transition at $D_{\mathrm{T}}=0.99$ as the damage zone keeps getting wider as no crack is introduced. This is illustrated in the following snapshots and this spurious damage zone extension was already identified in [6].

In order to study the effect of the CZM at the damage to crack transition, simulations in which the residual energy $\phi_{\mathrm{S}}$ is set to zero, i.e. the crack surfaces remain stress-free after the transition, are also carried out for $D_{\mathrm{T}}=0.95$ and for $D_{\mathrm{T}}=0.99$. However for a transition at $D_{\mathrm{T}}=0.95$, when such a stress-free crack initiates the loss of energy balance causes the simulation to diverge (no convergence of the Newton-Raphson iterations related to the non-local equations) and a dummy cohesive zone is thus considered with an energy to dissipate $\phi_{\mathrm{S}}^{*}$ equal to $20 \%$ of the correct energy $\phi_{\mathrm{S}}$ reported in Table 2 . The loading responses are reported in Fig. 6(b) where it can be seen that neglecting the remaining cohesive energy results in a sharp decrease of the loading curve followed by oscillations as compared to the corresponding consistent transition models which consider the correct energy $\phi_{\mathrm{S}}$ in the CZM. As expected, this sharp decrease is more pronounced when the crack initiates sooner for $D_{\mathrm{T}}=0.95$ as the missing energy is higher. This example demonstrates the requirement to introduce a CZM upon the damage to crack transition, for the numerical stability of the simulations, but also to respect the physics.

The damage distribution predicted by a simulation without crack introduction (pure CDM) and the damage predictions predicted by the simulations with two sets of transition parameters are successively illustrated for the relative loading pins displacements of 2, 3, 4 and $6 \mathrm{~mm}$ in Figs. 7, 8, 9 and 10, respectively. For $d=2 \mathrm{~mm}$, although the crack has already initiated for the lowest damage transition parameter, see Fig. 6(a), the crack opening remains small, see Figs. $7(\mathrm{c})$ and $7(\mathrm{~d})$, and the damage distribution remains similar as for the other set of transition parameters, Figs. $7(\mathrm{e})-7(\mathrm{f})$, and as when no crack is introduced, 


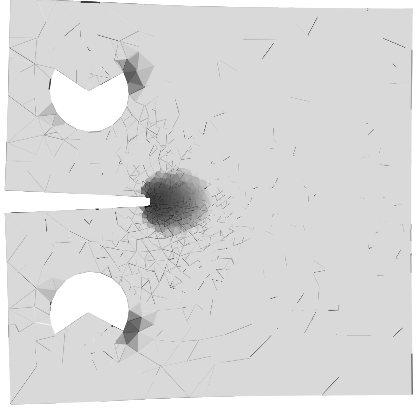

(a) Damage only

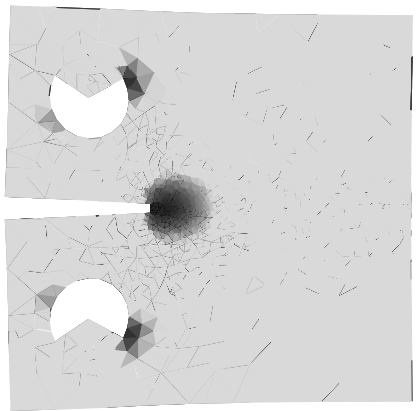

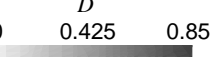

(c) $D_{\mathrm{T}}=0.85$
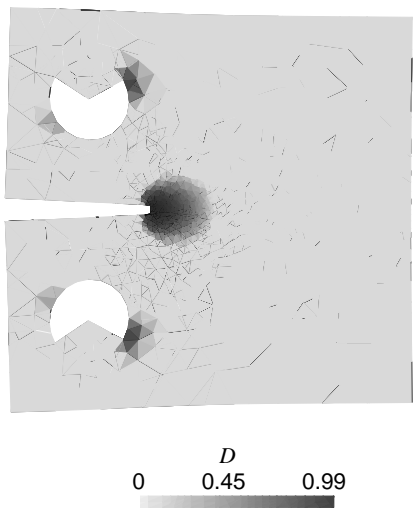

(e) $D_{\mathrm{T}}=0.99$

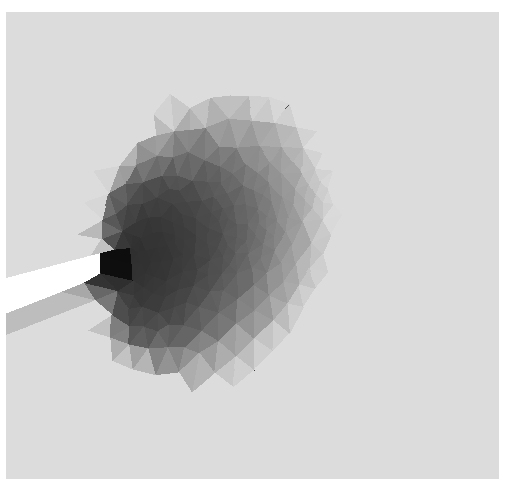

(b) Damage only

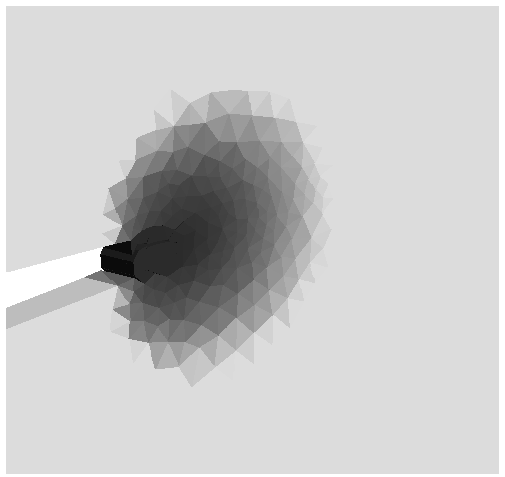

(d) $D_{\mathrm{T}}=0.85$

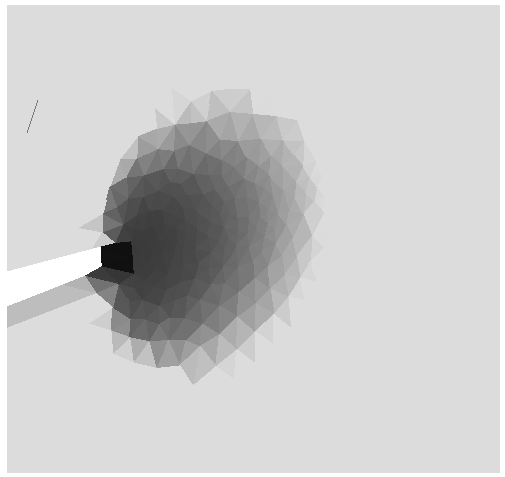

(f) $D_{\mathrm{T}}=0.99$

Figure 7: Damage representation in the Compact-Tension specimen at a pins-relative displacements $d=2 \mathrm{~mm}$ for the different transition parameters. Right column: Zoom around the notched area.

Figs. 7(a)-7(b) -note that the scales are different explaining why the damage zone seems slightly wider for the $D_{\mathrm{T}}=0.85$. Because of the non-local approach the damage does not localize to single elements but is distributed around the notch. For a relative pins displacement $d=3 \mathrm{~mm}$, the crack has propagated for the lower damage transition parameters and has just initiated for the higher one, see Figs. 8(c)-8(f). One more time the damage zone is comparable for the three simulations. During the structural softening stage at $d=4 \mathrm{~mm}$, Fig. 9, and at $d=6 \mathrm{~mm}$, Fig. 10, the crack keeps propagating with a further extension 


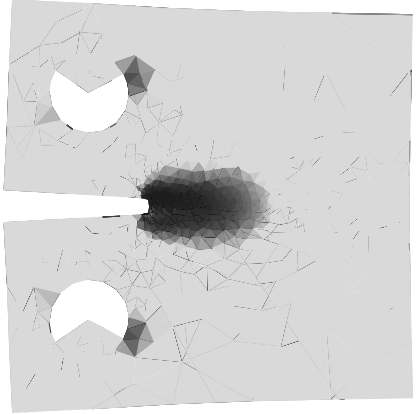

$\begin{array}{ccc} & D & \\ 0 & 0.5 & 1 .\end{array}$

(a) Damage only

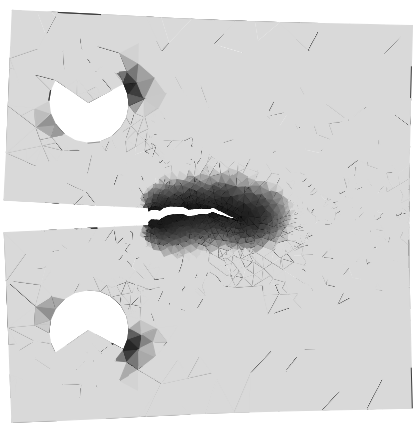

$\begin{array}{lll}0 & 0.425 & 0.85\end{array}$

(c) $D_{\mathrm{T}}=0.85$

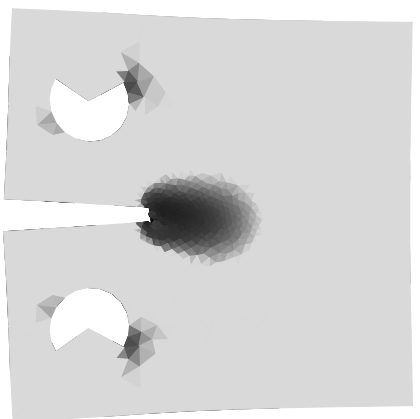

$\begin{array}{ccc} & D & \\ 0 & 0.45 & 0.99\end{array}$

(e) $D_{\mathrm{T}}=0.99$

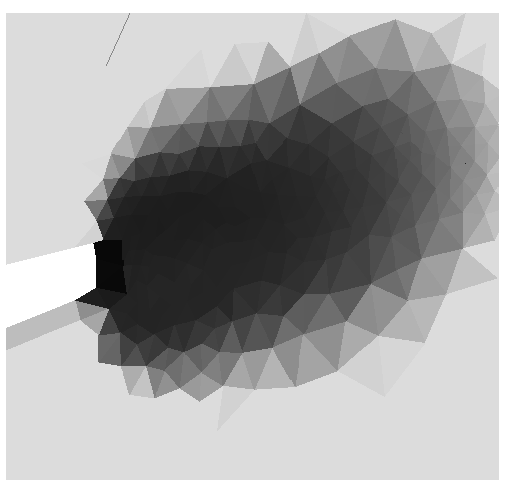

(b) Damage only

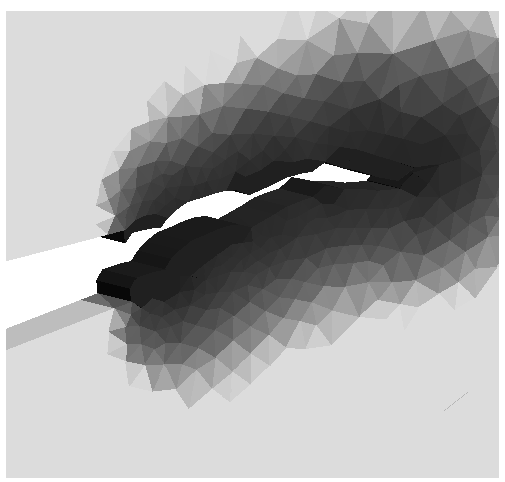

(d) $D_{\mathrm{T}}=0.85$

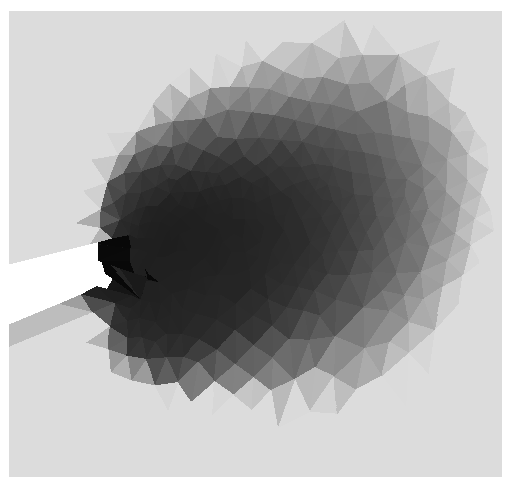

(f) $D_{\mathrm{T}}=0.99$

Figure 8: Damage representation in the Compact-Tension specimen at a pins-relative displacements $d=3 \mathrm{~mm}$ for the different transition parameters. Right column: Zoom around the notched area.

for the lowest damage transition parameter $D_{\mathrm{T}}=0.85$. However both the crack opening and the damage zones remain comparable for the three simulations. Note that when allowing damage to reach high values, one element is distorted due to the high value of damage reached, but without impeding on the numerical results. Such a detachment of a single element is common with the use of the cohesive zone method and is known as "dust element". 

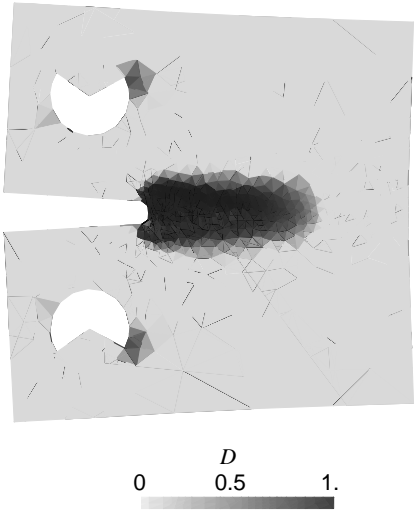

(a) Damage only

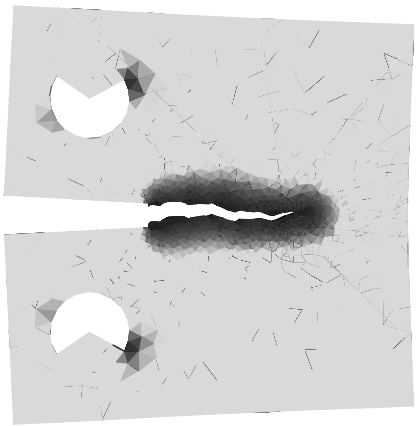

$\begin{array}{ccc} & D & \\ 0 & 0.425 & 0.85\end{array}$

(c) $D_{\mathrm{T}}=0.85$
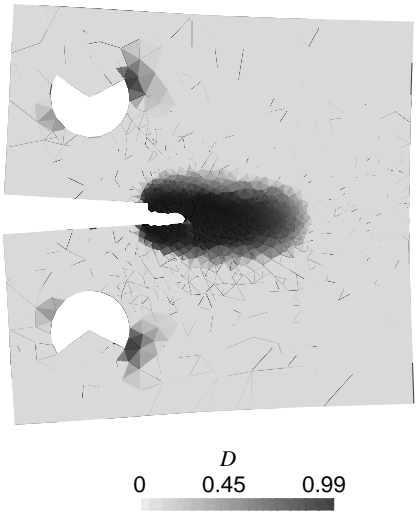

(e) $D_{\mathrm{T}}=0.99$

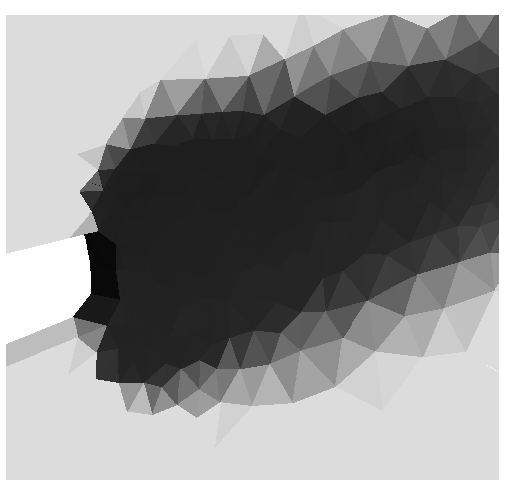

(b) Damage only

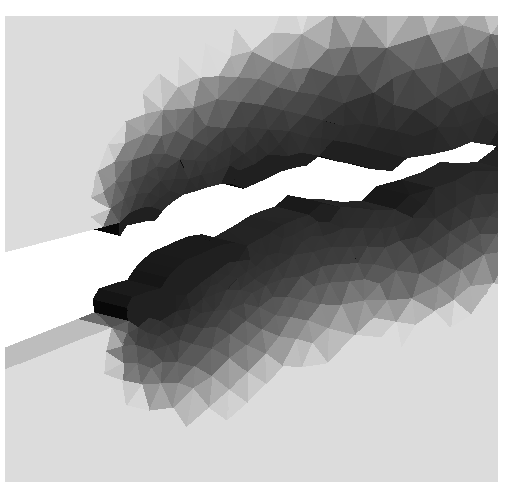

(d) $D_{\mathrm{T}}=0.85$

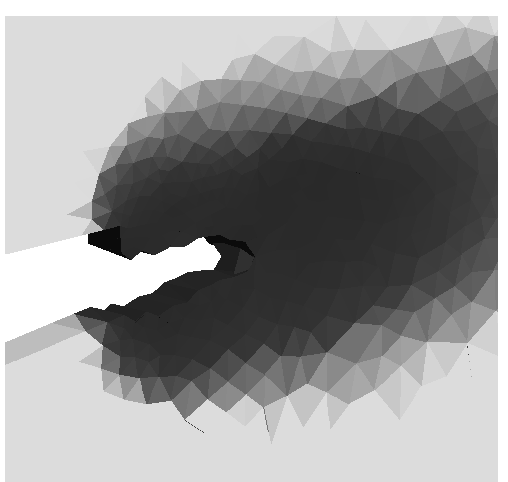

(f) $D_{\mathrm{T}}=0.99$

Figure 9: Damage representation in the Compact-Tension specimen at a pins-relative displacements $d=4$ mm for the different transition parameters. Right column: Zoom around the notched area.

\subsection{Double-Notched specimen}

The Double-Notched specimen (DNS) is now considered to assess the initiation of multiple cracks and the mesh dependency. The geometry and the boundary conditions of the specimen are illustrated in Fig. 11(a), and the dimensions are $a=50 \mathrm{~mm}, r_{c}=5 \mathrm{~mm}, r_{1}=10 \mathrm{~mm}, r_{2}=12.5 \mathrm{~mm}$, and thickness $t=5 \mathrm{~mm}$.

The material is the same as for the CTS test with the properties and the parameters summarized in 


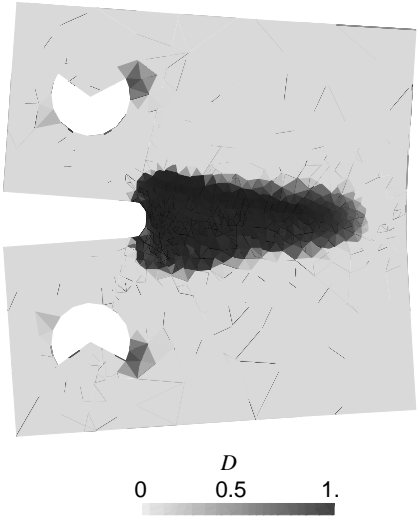

(a) Damage only

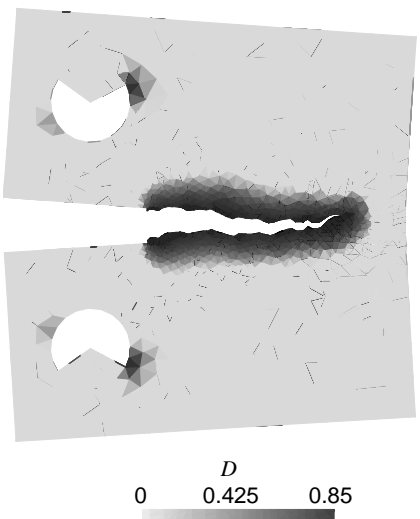

(c) $D_{\mathrm{T}}=0.85$

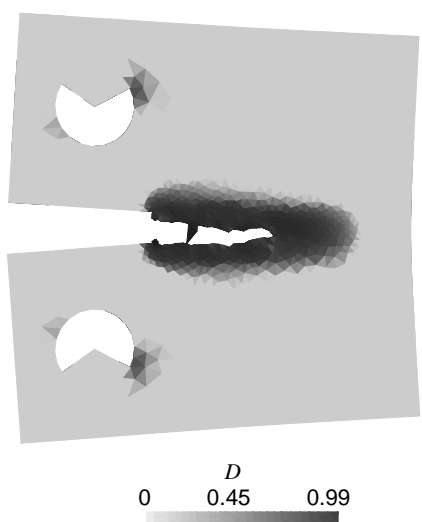

(e) $D_{\mathrm{T}}=0.99$

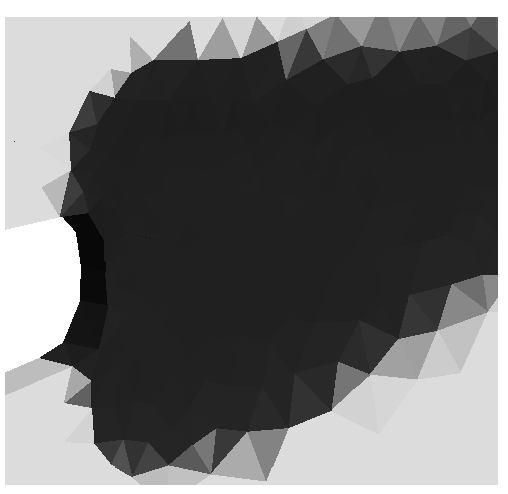

(b) Damage only

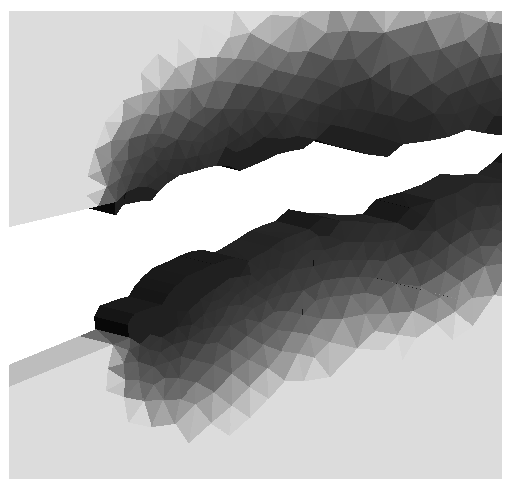

(d) $D_{\mathrm{T}}=0.85$

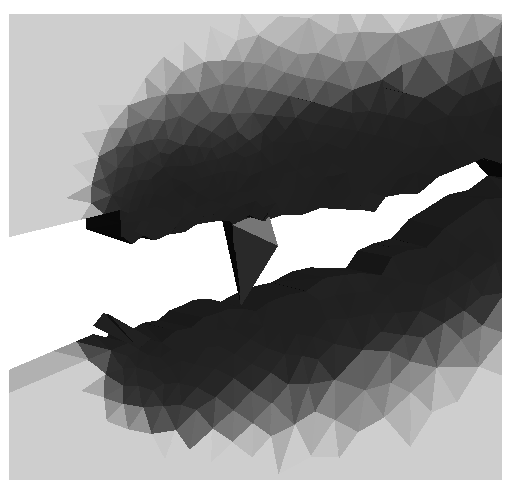

(f) $D_{\mathrm{T}}=0.99$

Figure 10: Damage representation in the Compact-Tension specimen at a pins-relative displacements $d=6 \mathrm{~mm}$ for the different transition parameters. Right column: Zoom around the notched area.

Table 1. The 3D finite element model of half of the specimen ${ }^{7}$. The mesh is partitioned on 64 processors. Different meshes are successively considered. A coarse mesh with 10300 quadratic 10-node tetrahedra with a mesh size at the notches equal to $0.8 \mathrm{~mm}$ as illustrated in Fig. 11(b), a medium mesh of 34500 quadratic

\footnotetext{
${ }^{7}$ For symmetry reasons only half of the thickness is considered
} 


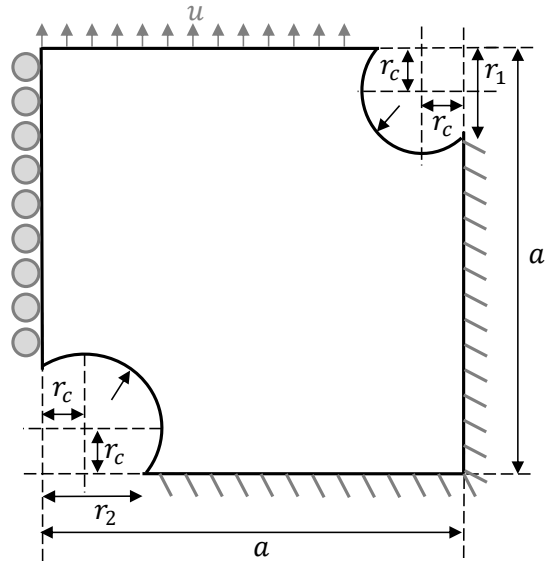

(a) Geometry

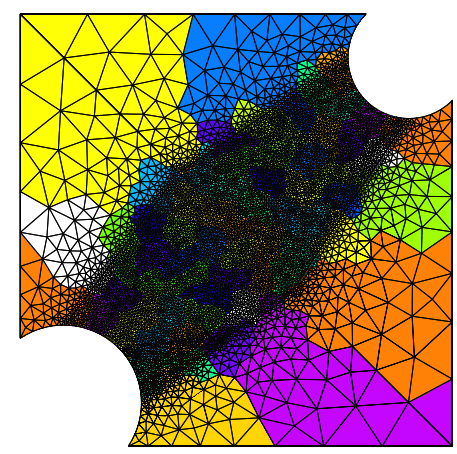

(c) Medium Mesh

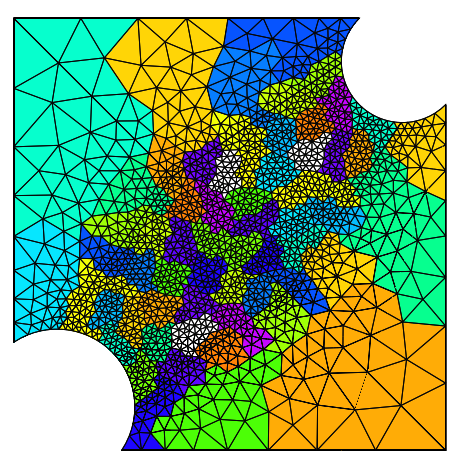

(b) Coarse Mesh

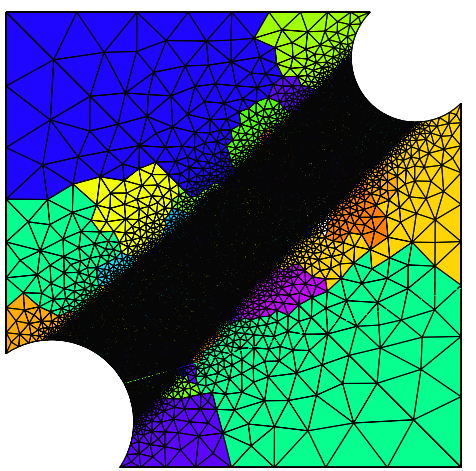

(d) Fine Mesh

Figure 11: The Double-Notched specimen. (a) Geometry and boundary conditions with $a=50 \mathrm{~mm}, r_{c}=5 \mathrm{~mm}, r_{1}=10 \mathrm{~mm}$, $r_{2}=12.5 \mathrm{~mm}$, and thickness $t=5 \mathrm{~mm}$. (b) Partitioned coarse mesh, (c) medium mesh, and (d) fine mesh, with the different partitions in different colors.

10-node tetrahedra with the element size at the notches equal to $0.4 \mathrm{~mm}$ as illustrated in Fig. 11(c), and a fine mesh of 53291 quadratic 10-node tetrahedra with the element size at the notches equal to $0.3 \mathrm{~mm}$ as illustrated in Fig. 11(c). A finer discretization is always applied in the band where the cracks are expected to initiate and propagate, while a coarse mesh is used far away from this damage process zone. Crack transition is set for a corresponding damage equal to $D_{\mathrm{T}}=0.85$.

The evolutions of the force with respect to the boundary displacement is reported in Fig. 12 for the three mesh sizes. As it can be seen a decrease in the stiffness of the sample happens around $u=0.5 \mathrm{~mm}$ and the crack appears for a displacement of $u=0.65 \mathrm{~mm}$. The result remains insensitive from the mesh size, even during the softening stage of the response. The only difference that can be seen are numerical oscillations, resulting from the time-integration, which increase with the mesh refinement.

The deformations obtained for the medium mesh of the DNS and for an upper boundary displacement of $0.5 \mathrm{~mm}$ are illustrated in Fig. 13. The damage distribution remains confined to the notch areas and the displacement distribution shows a regular gradient. However for a boundary displacement of $u=1$ $\mathrm{mm}$ the damaged zone has extended between the two notches, see Fig. 14 and the displacement now 


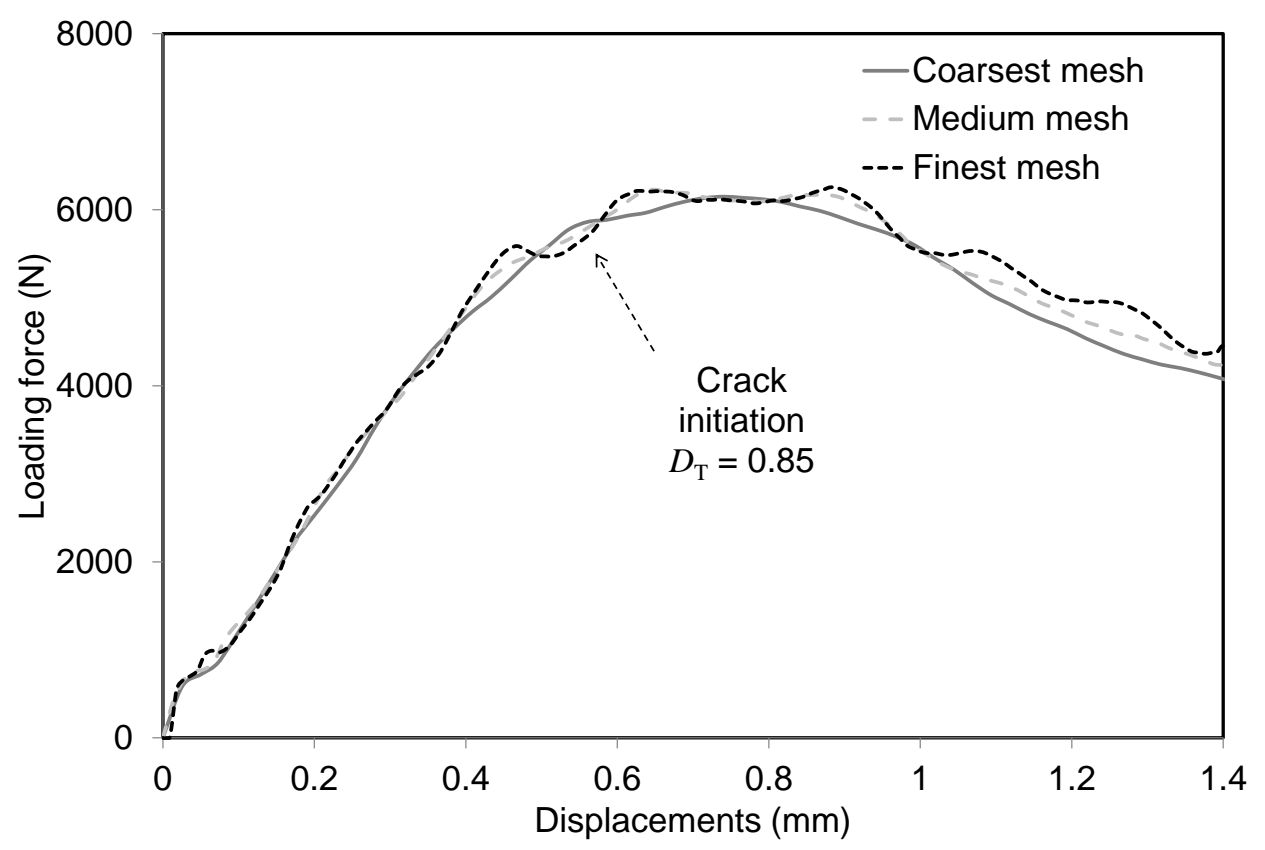

Figure 12: Force vs. displacement curves obtained for the Double-Notched specimen.

exhibits a localization in that zone, see Fig. 14(a), with an almost constant displacement on each side of the localization band. For this boundary displacement the cracks have initiated at both notches. For a boundary displacement of $u=1.5 \mathrm{~mm}$, the two cracks have propagated within the localization zone, see Fig. 15. Additional cracks have appeared at the center of the specimen within the localization band. As the mesh is fine the alignement of these cracks is close to the direction of this localization band as there exist enough possibilities for the cohesive elements to open.

\section{Conclusions}

In this work the hybrid DG/extrinsic cohesive law framework is extended to the damage to crack challenge.

The simulations start considering a non-local damage material model implemented following a DG approach. Such a regularized damage model allows complex degradation processes to be simulated without suffering from a mesh dependence issue. However with such a CDM model, large element distortions arise as a result of the stress-carrying capacity loss of the constitutive material response, which disagree with the physical reality but also hurt the numerical convergence of the simulation.

The CDM model is thus locally substituted by a CZM to introduce a crack discontinuity. The construction of an energetically consistent TSL in the CZM is based on the theoretical energy equivalence between the non-local damage mechanics continuous model and the discontinuous model for a $1 \mathrm{D}$ setting. In this paper only the case of elastic damage is considered and a simple transition criterion based on the effective stress is developed. This criterion is applied at the interface elements and the direction of the crack propagation is thus only governed by the structural response, i.e. no direction criterion is required.

The transition between the CDM and the CZM is made possible and computationally efficient in a parallel framework because of the DG formulation of the non-local damage model. Indeed because of the existence of interface elements since the very beginning of the simulation and of the consistency of the DG model the limitations of usual CZM are avoided, i.e. either the difficulty of inserting them on-the-fly with the extrinsic method or the modification of structure compliance with the intrinsic method.

The method was validated on the CTS test by comparing the effect of the transition criterion. It was found that the peak value obtained for the structural response is independant from the damage value at 


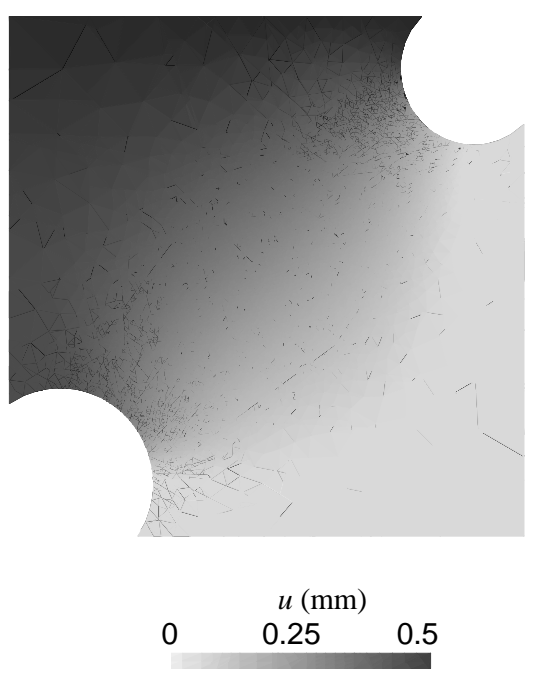

(a) Displacement

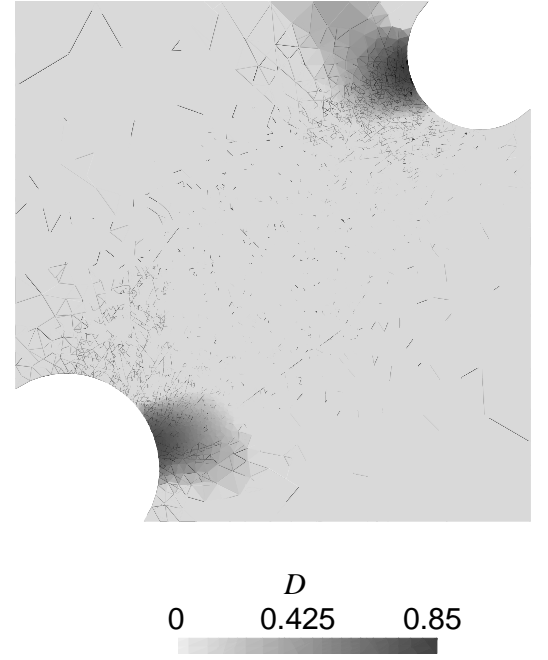

(b) Damage

Figure 13: Double-notched specimen with a medium discretization and for a boundary displacement of 0.5 mm: (a) Displacement field, (b) Damage distribution.

which the crack is inserted. The begining part of the softening reponse was slightly lower when introducing the crack earlier as the compliance of the structure increases. However the tail of the response was found to be insensitive of the damage to crack transition. The introduction of the crack prevents the artificial extension of the damage zone arising with a pure non-local damage approach.

The effect of the mesh size was studied on the DNS. The initiation of a crack at both notches was captured for all the mesh sizes considered and the structural response was found to be independant of this mesh size.

The current limitations of the work are the small deformations setting and the elastic-damage assumption. In a future work it is intended to develop a large deformation formalism considering more complex damage models. This will require to extend the hybrid DG/ECL formalism, but also to define new energetic equivalence models between the more developed CDM and the CZM. Moreover when considering ductile materials, it might be necessary to introduce the crack for lower values of the damage, as this is observed experimetally. Thus the cohesive model will have to consider the stress triaxiality effect by accounting for the in-plane stretch as proposed in $[49,51]$.

\section{Acknowledgment}

Computational resources have been provided by the supercomputing facilities of the "Consortium des Équipements de Calcul Intensif en Fédération Wallonie Bruxelles (CÉCI)" funded by the "Fond de la Recherche Scientifique de Belgique (FRS-FNRS)". The project was funded by the "Féération Wallonie Bruxelles" through a ULg starting grant.

\section{Appendix A. Linearization of the elastic-damage model}

From (11-13) one has directly

$$
\begin{aligned}
\delta \boldsymbol{\sigma} & =\left[(1-D) \frac{\partial \hat{\boldsymbol{\sigma}}}{\partial \boldsymbol{\varepsilon}}-\hat{\boldsymbol{\sigma}} \otimes \frac{\partial D}{\partial \boldsymbol{\varepsilon}}\right]: \delta \boldsymbol{\varepsilon}-\hat{\boldsymbol{\sigma}} \frac{\partial D}{\partial \tilde{e}} \delta \tilde{e} \\
& =\underbrace{\left[(1-D) \mathbb{C}-\hat{\boldsymbol{\sigma}} \otimes \frac{\partial D}{\partial \boldsymbol{\varepsilon}}\right]}_{\mathbb{C}_{\boldsymbol{u} \boldsymbol{u}}}: \delta \boldsymbol{\varepsilon}-\underbrace{\hat{\boldsymbol{\sigma}} \frac{\partial D}{\partial \tilde{e}}}_{\boldsymbol{C}_{\boldsymbol{u} \tilde{e}}} \delta \tilde{e},
\end{aligned}
$$



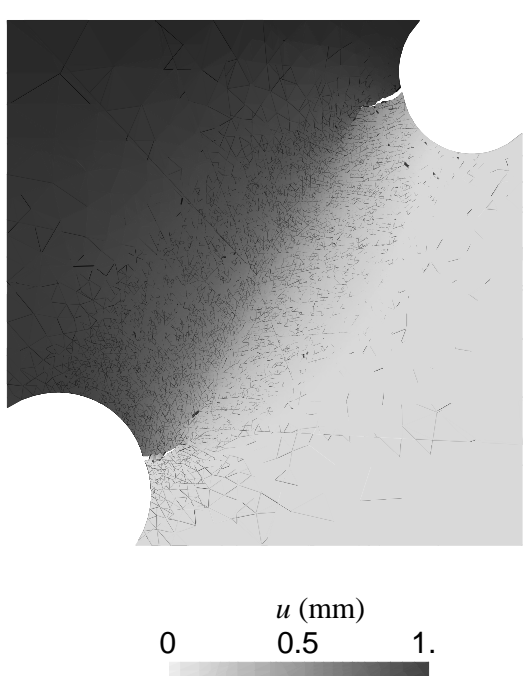

(a) Displacement
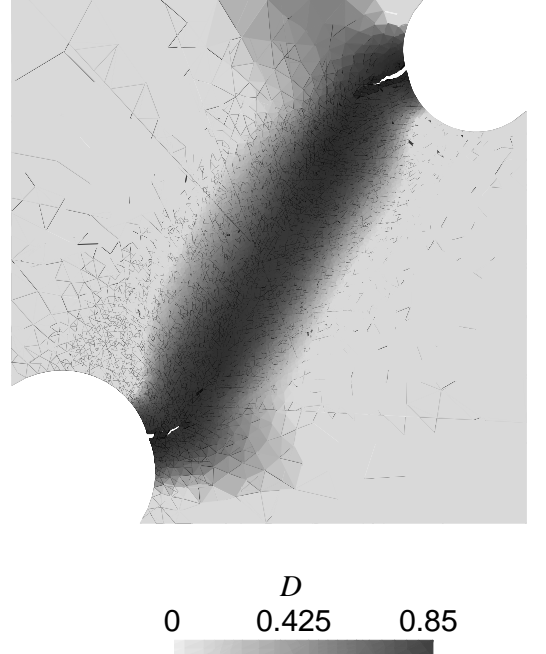

(b) Damage

Figure 14: Double-notched specimen with a medium discretization and for a boundary displacement of $1 \mathrm{~mm}$ : (a) Displacement field, (b) Damage distribution.

where the missing terms $\frac{\partial D}{\partial \varepsilon}$ and $\frac{\partial D}{\partial \tilde{e}}$ can be derived from the applied damage model. Finally, the linearization of the local equivalent strain $e$ reads

$$
\delta e=\frac{\partial e}{\partial \varepsilon} \cdot \delta \varepsilon=\boldsymbol{C}_{e \boldsymbol{u}} \cdot \delta \boldsymbol{\varepsilon},
$$

where $\frac{\partial e}{\partial \varepsilon}$ depends on the definition of the chosen equivalent strain.

\section{Appendix B. Formulation of the finite element forces}

The weak formulation (65-66) of the non-local implicit DG/ECL framework is taken as the basis for the finite element discretization. Applying the discretization (69-70) in the weak form (65-66) leads directly to the following expressions of the elementary (bulk elements $\Omega^{e}$ and interface elements $\Gamma_{\mathrm{I}}^{s}$ ) nodal forces

$$
\begin{aligned}
\boldsymbol{f}_{\boldsymbol{u} \text { int }}^{e{ }^{a}=} & \int_{\Omega^{e}} \boldsymbol{\sigma} \cdot \boldsymbol{\nabla} N^{a} d V \\
\boldsymbol{f}_{\boldsymbol{u} \text { ext }}^{e}{ }^{a}= & \int_{\Omega^{e}} \rho \boldsymbol{b} N^{a} d V+\int_{\Gamma_{\mathrm{N}}} N^{a} \cdot \overline{\boldsymbol{t}} d s \\
\boldsymbol{f}_{\boldsymbol{u} \mathrm{I}}^{s a^{ \pm}}= & \pm \int_{\Gamma_{\mathrm{IC}}^{s}} \overline{\boldsymbol{t}}^{-}(\llbracket \boldsymbol{u} \rrbracket) N^{a^{ \pm}} d s \pm \int_{\Gamma_{\mathrm{IU}}^{s}}\langle\boldsymbol{\sigma}\rangle \cdot \boldsymbol{n}^{-} N^{a^{ \pm}} d s+ \\
& \frac{1}{2} \int_{\Gamma_{\mathrm{IU}}^{s}} \nabla N^{a^{ \pm}} \cdot\left[\left(\llbracket \boldsymbol{u} \rrbracket \otimes \boldsymbol{n}^{-}\right): \mathbb{C}^{ \pm}\right] d s \pm \int_{\Gamma_{\mathrm{IU}}^{s}}\left[\left\langle\frac{\beta_{s}}{h_{s}} \mathbb{C}\right\rangle: \llbracket \boldsymbol{u} \rrbracket \otimes \boldsymbol{n}^{-}\right] \cdot \boldsymbol{n}^{-} N^{a^{ \pm}} d s, \\
f_{\tilde{e} \text { int }}^{e}{ }^{a}= & \int_{\Omega^{e}}\left(\tilde{e} N^{a}+\nabla N^{a} \cdot c \boldsymbol{\nabla} \tilde{e}\right) d V, \\
f_{e \text { int }}^{e}{ }^{a}= & \int_{\Omega^{e}} e N^{a} d V, \text { and } \\
f_{\tilde{e} \mathrm{IU}}^{s} a^{ \pm}= & \pm \int_{\Gamma_{\mathrm{I}}^{s}}\langle c \boldsymbol{\nabla} \tilde{e}\rangle \cdot \boldsymbol{n}^{-} N^{a^{ \pm}} d s+\frac{1}{2} \int_{\Gamma_{\mathrm{IU}}^{s}} \llbracket \tilde{e} \rrbracket \nabla N^{a^{ \pm}} \cdot c^{ \pm} \boldsymbol{n}^{-} d s \pm \\
& \int_{\Gamma_{\mathrm{IU}}^{s}} \boldsymbol{n}^{-} \cdot\left\langle\frac{\beta_{s}}{h_{s}} c\right\rangle \cdot \boldsymbol{n}^{-} \llbracket \tilde{e} \rrbracket N^{a^{ \pm}} d s .
\end{aligned}
$$




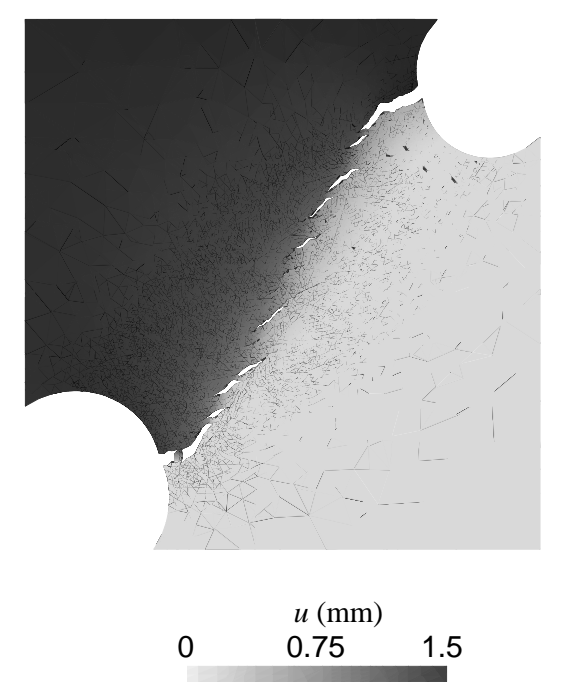

(a) Displacement

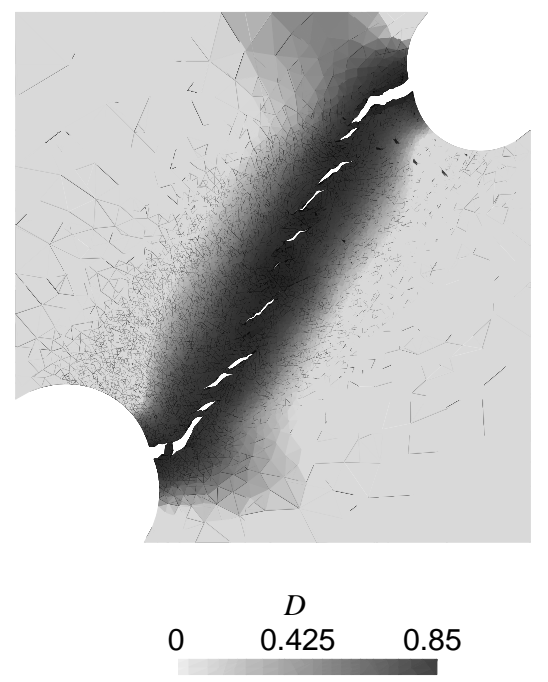

(b) Damage

Figure 15: Double-notched specimen with a medium discretization and for a boundary displacement of 1.5 mm: (a) Displacement field, (b) Damage distribution.

The interface forces arising from the DG/ECL formulation are evaluated from the shape functions $N^{a^{+}}$ and $N^{a^{-}}$of the + and - elements sharing the same interface and the same nodes $a$, although the degrees of freedom at the same node for the two elements are distinct. This is a particularity of the presented implementation, which duplicates the degrees of freedom at a common node instead of duplicating the nodes at common interfaces as this is usually done for cohesive methods.

In the integration above the shape functions $N^{a}$ explicitly used are the ones of the volume elements and they are evaluated at the integration points of the interface elements. Due to the symmetrization terms all the nodes of the 2 neighboring tetrahedra have force contributions, and not only the nodes of the common interface. The evaluation of the interface forces requires a full 6-point integration at the interface element in order to avoid spurious penetration modes as shown in [54].

The linearization of the elementary forces (B.1-B.6) can be easily obtained using (A.1) and (A.2) as

$$
\begin{aligned}
& K_{\boldsymbol{u u} \text { int } i k}^{e b}=\frac{\partial f_{\boldsymbol{u} \text { int } i}^{a}}{\partial u_{k}^{b}}=\int_{\Omega^{e}} \nabla_{j} N^{a} \mathbb{C}_{\boldsymbol{u u} i j k l} \nabla_{l} N^{b} d V, \\
& K_{\boldsymbol{u} \tilde{e} \operatorname{int} i}^{e}{ }^{a b}=\frac{\partial f_{\boldsymbol{u}}^{e}{ }_{\operatorname{int} i}^{a}}{\partial \tilde{\mathbf{e}}^{b}}=\int_{\Omega^{e}} \nabla_{j} N^{a} C_{\boldsymbol{u} \tilde{e} i j} N^{b} d V, \\
& K_{\boldsymbol{u} \boldsymbol{u} \mathrm{I} i k}^{a^{ \pm} b^{ \pm}}=\frac{\partial f_{\boldsymbol{u} \mathrm{I} i}^{s a^{ \pm}}}{\partial u_{k}^{b^{ \pm}}}= \pm \pm \int_{\Gamma_{\mathrm{IC}}^{s}} \frac{\partial \bar{t}_{i}^{-}}{\partial \llbracket u \rrbracket_{k}} N^{a^{ \pm}} N^{b^{ \pm}} d s \\
& \pm+\frac{1}{2} \int_{\Gamma_{\mathrm{IU}}^{s}} \mathbb{C}_{\text {uu }}^{ \pm}{ }_{i j k l} n_{j}^{-} N^{a^{ \pm}} \nabla_{l} N^{b^{ \pm}} d s \\
& + \pm \frac{1}{2} \int_{\Gamma_{\mathrm{IU}}^{s}} \nabla_{j} N^{a^{ \pm}} N^{b^{ \pm}} n_{l}^{-} \mathbb{C}_{k l i j}^{ \pm} d s \\
& \pm \pm \int_{\Gamma_{\mathrm{IU}}^{s}}\left\langle\frac{\beta_{s}}{h_{s}} \mathbb{C}_{i j k l}\right\rangle n_{l}^{-} n_{j}^{-} N^{a^{ \pm}} N^{b^{ \pm}} d s \\
& K_{\boldsymbol{u} \tilde{e} \mathrm{I} i}^{s a^{ \pm} b^{ \pm}}=\frac{\partial f_{\boldsymbol{u} \mathrm{I} i}^{s a^{ \pm}}}{\partial \tilde{\mathbf{e}}^{b^{ \pm}}}= \pm+\frac{1}{2} \int_{\Gamma_{\mathrm{IU}}^{s}} C_{\boldsymbol{u} \tilde{e} i j}^{ \pm} n_{j}^{-} N^{a^{ \pm}} N^{b^{ \pm}} d s,
\end{aligned}
$$


where the notation " \pm " means the consecutive application of " \pm " and " \pm ", and

$$
\begin{aligned}
& K_{\tilde{e} \boldsymbol{u} \text { int } k}^{e}{ }^{a b}=\frac{\partial f_{\tilde{e} \text { int }}^{e}}{\partial u_{k}^{b}}=0, \\
& K_{\tilde{e} \tilde{e} \mathrm{int}}^{e}{ }^{a b}=\frac{\partial f_{\tilde{e} \mathrm{int}}^{e}{ }^{a}}{\partial \tilde{e}^{b}}=\int_{\Omega^{e}}\left(N^{a} N^{b}+\nabla_{i} N^{a} c_{\mathrm{g}_{i j}} \nabla_{j} N^{b}\right) d V, \\
& K_{e \boldsymbol{u} \text { int } k}^{e b}=\frac{\partial f_{e \text { int }}^{e}}{\partial u_{k}^{b}}=\int_{\Omega^{e}} C_{e \boldsymbol{u} k l} N^{a} \nabla_{l} N^{b} d V, \\
& K_{e \tilde{e} \text { int }}^{e}{ }^{a b}=\frac{\partial f_{e \text { int }}^{e}{ }^{a}}{\partial \tilde{e}^{b}}=0,
\end{aligned}
$$

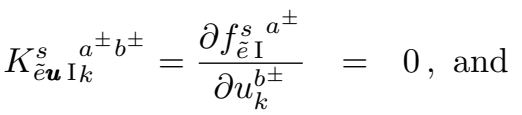

$$
\begin{aligned}
& K_{\tilde{e} \tilde{e} \mathrm{I}}^{s} a^{ \pm} b^{ \pm}=\frac{\partial f_{\tilde{e} \mathrm{I}}^{s} a^{ \pm}}{\partial \tilde{e}^{b^{ \pm}}}= \pm+\frac{1}{2} \int_{\Gamma_{\mathrm{IU}}^{s}} n_{i}^{-} c^{ \pm} \nabla_{i} N^{b^{ \pm}} N^{a^{ \pm}} d s+ \pm \int_{\Gamma_{\mathrm{IU}}^{s}} \frac{1}{2} \nabla_{i} N^{a^{ \pm}} c^{ \pm} n_{i}^{-} N^{b^{ \pm}} d s \\
& \pm \pm \int_{\Gamma_{\mathrm{IU}}^{s}} n_{i}^{-}\left\langle\frac{\beta_{s}}{h_{s}} c\right\rangle n_{i}^{-} N^{a^{ \pm}} N^{b^{ \pm}} d s
\end{aligned}
$$

\section{References}

[1] J. Lemaitre, J.-L. Chaboche, Mécanique des Matériaux solides, Dunod, ISBN 2040157867, 1991.

[2] J. Lemaitre, Coupled elasto-plasticity and damage constitutive equations, Computer Methods in Applied Mechanics and Engineering 51 (1-3) (1985) 31 - 49, ISSN 0045-7825, doi:DOI: 10.1016/0045-7825(85)90026-X.

[3] I. Doghri, Numerical implementation and analysis of a class of metal plasticity models coupled with ductile damage, International Journal for Numerical Methods in Engineering 38 (20) (1995) 3403-3431, ISSN 1097-0207, doi: $10.1002 /$ nme.1620382004.

[4] Z. P. Bažant, T. B. Belytchko, T. P. Chang, Continuum Theory for Strain-Softening, Journal of Engineering MechanicsASCE 110 (12) (1984) 1666-1692, doi:10.1061/(ASCE)0733-9399(1984)110:12(1666).

[5] R. Peerlings, R. de Borst, W. Brekelmans, S. Ayyapureddi, Gradient-enhanced damage for quasi-brittle materials, International Journal for Numerical Methods in Engineering 39 (1996) 3391-3403.

[6] M. Geers, Experimental Analysis and Computational Modelling of Damage and Fracture, Ph.D. thesis, University of Technology, Eindhoven (Netherlands)., 1997.

[7] R. Peerlings, R. de Borst, W. Brekelmans, M. Geers, Gradient-enhanced damage modelling of concrete fracture, Mechanics of Cohesive-Frictional Materials 3 (1998) 323-342.

[8] R. Peerlings, R. de Borst, W. Brekelmans, M. Geers, Localisation issues in local and nonlocal continuum approaches to fracture, Eur. J. Mech. A/Solids 21 (2002) 175-189.

[9] H. M. Zbib, E. C. Aifantis, A Gradient-Dependent Flow Theory of Plasticity: Application to Metal and Soil Instabilities, Applied Mechanics Reviews 42 (11S) (1989) S295-S304, doi:10.1115/1.3152403.

[10] G. Barenblatt, The Mathematical Theory of Equilibrium Cracks in Brittle Fracture, vol. 7, Elsevier, 55-129, doi: 10.1016/S0065-2156(08)70121-2, 1962

[11] D. S. Dugdale, Yielding of steel sheets containing slits, Journal of the Mechanics and Physics of Solids 8 (2) (1960) 100-104, ISSN 0022-5096.

[12] N. Moës, J. Dolbow, T. Belytschko, A finite element method for crack growth without remeshing, International Journal for Numerical Methods in Engineering 46 (1) (1999) 131-150, ISSN 1097-0207, doi:10.1002/(SICI)10970207(19990910)46:1<131::AID-NME726>3.0.CO;2-J.

[13] N. Moës, T. Belytschko, Extended finite element method for cohesive crack growth, Engineering Fracture Mechanics 69 (7) (2002) 813-833, ISSN 0013-7944, doi:10.1016/S0013-7944(01)00128-X.

[14] F. Armero, C. Linder, Numerical simulation of dynamic fracture using finite elements with embedded discontinuities, International Journal of Fracture 160 (2009) 119-141, ISSN 0376-9429, doi:10.1007/s10704-009-9413-9.

[15] R. de Borst, M. A. Gutiérrez, G. N. Wells, J. J. C. Remmers, H. Askes, Cohesive-zone models, higher-order continuum theories and reliability methods for computational failure analysis, International Journal for Numerical Methods in Engineering 60 (1) (2004) 289-315, ISSN 1097-0207, doi:10.1002/nme.963.

[16] A. Hillerborg, M. Modéer, P.-E. Petersson, Analysis of crack formation and crack growth in concrete by means of fracture mechanics and finite elements, Cement and Concrete Research 6 (6) (1976) 773 - 781, ISSN 0008-8846, doi:10.1016/00088846(76)90007-7.

[17] A. Needleman, A continuum model for void nucleation by inclusion debonding, Journal of Applied Mechanics 54 (1987) $525-531$.

[18] V. Tvergaard, Effect of fibre debonding in a whisker-reinforced metal, Materials Science and Engineering: A 125 (2) (1990) 203 - 213, ISSN 0921-5093, doi:10.1016/0921-5093(90)90170-8. 
[19] X.-P. Xu, A. Needleman, Numerical simulations of fast crack growth in brittle solids, Journal of the Mechanics and Physics of Solids 42 (9) (1994) 1397 - 1434, ISSN 0022-5096, doi:10.1016/0022-5096(94)90003-5.

[20] G. T. Camacho, M. Ortiz, Computational modelling of impact damage in brittle materials, International Journal of Solids and Structures 33 (20-22) (1996) 2899-2938, ISSN 0020-7683.

[21] A. Pandolfi, P. Guduru, M. Ortiz, A. Rosakis, Three dimensional cohesive-element analysis and experiments of dynamic fracture in C300 steel, International Journal of Solids and Structures 37 (27) (2000) 3733 - 3760, ISSN 0020-7683, doi: 10.1016/S0020-7683(99)00155-9.

[22] J. Mergheim, E. Kuhl, P. Steinmann, A hybrid discontinuous Galerkin/interface method for the computational modelling of failure, Communications in Numerical Methods in Engineering 20 (7) (2004) 511-519, URL http://dx.doi.org/10.1002/cnm.689.

[23] R. Radovitzky, A. Seagraves, M. Tupek, L. Noels, A scalable 3D fracture and fragmentation algorithm based on a hybrid, discontinuous Galerkin, cohesive element method, Computer Methods in Applied Mechanics and Engineering 200 (2011) 326-344, ISSN 0045-7825, doi:10.1016/j.cma.2010.08.014.

[24] M. Prechtel, G. Leugering, P. Steinmann, M. Stingl, Towards optimization of crack resistance of composite materials by adjustment of fiber shapes, Engineering Fracture Mechanics 78 (6) (2011) 944 - 960, ISSN 0013-7944, doi: 10.1016/j.engfracmech.2011.01.007.

[25] L. Wu, D. Tjahjanto, G. Becker, A. Makradi, A. Jérusalem, L. Noels, A micromeso-model of intra-laminar fracture in fiber-reinforced composites based on a discontinuous Galerkin/cohesive zone method, Engineering Fracture Mechanics 104 (2013) 162-183, ISSN 0013-7944, doi:10.1016/j.engfracmech.2013.03.018.

[26] G. Becker, C. Geuzaine, L. Noels, A one Field Full Discontinuous Galerkin Method for Kirchhoff-Love Shells Applied to Fracture Mechanics, Computer Methods in Applied Mechanics and Engineering 200 (2011) 3223-3241, ISSN 0045-7825, doi:10.1016/j.cma.2011.07.008.

[27] G. Becker, L. Noels, A full discontinuous Galerkin formulation of non-linear Kirchhoff-Love shells: elasto-plastic finite deformations, parallel computation \& fracture applications, International Journal for Numerical Methods in Engineering 93 (2013) 80-117, ISSN 1097-0207, doi:10.1002/nme.4381.

[28] J. Mediavilla, R. H. J. Peerlings, M. G. D. Geers, Discrete crack modelling of ductile fracture driven by non-local softening plasticity, International Journal for Numerical Methods in Engineering 66 (4) (2006) 661-688, ISSN 1097-0207, doi: $10.1002 /$ nme.1572.

[29] J. Mediavilla, R. Peerlings, M. Geers, An integrated continuous-discontinuous approach towards damage engineering in sheet metal forming processes, Engineering Fracture Mechanics 73 (7) (2006) 895-916.

[30] M. R. R. Seabra, J. M. C. Sa, F. X. Andrade, F. F. Pires, Continuous-discontinuous formulation for ductile fracture, International Journal of Material Forming 4 (3) (2011) 271-281, ISSN 1960-6206, doi:10.1007/s12289-010-0991-x.

[31] M. R. Seabra, P. Šuštarič, J. M. Cesar de Sa, T. Rodič, Damage driven crack initiation and propagation in ductile metals using XFEM, Computational Mechanics 52 (1) (2013) 161-179, ISSN 0178-7675, doi:10.1007/s00466-012-0804-9.

[32] A. Simone, G. N. Wells, L. J. Sluys, From continuous to discontinuous failure in a gradient-enhanced continuum damage model, Computer Methods in Applied Mechanics and Engineering 192 (4142) (2003) 4581 - 4607, ISSN 0045-7825, doi: 10.1016/S0045-7825(03)00428-6.

[33] H. Huespe, A. Needleman, J. Oliver, P. Sánchez, A finite thickness band method for ductile fracture analysis, International Journal of Plasticity 25 (12) (2009) 2349 - 2365, ISSN 0749-6419, doi:10.1016/j.ijplas.2009.03.005.

[34] N. Moës, C. Stolz, P.-E. Bernard, N. Chevaugeon, A level set based model for damage growth: The thick level set approach, International Journal for Numerical Methods in Engineering 86 (3) (2011) 358-380, ISSN 1097-0207, doi:10.1002/nme.3069.

[35] J. Planas, M. Elices, G. Guinea, Cohesive cracks versus nonlocal models: Closing the gap, International Journal of Fracture 63 (2) (1993) 173-187, ISSN 0376-9429, doi:10.1007/BF00017284.

[36] J. Mazars, G. Pijaudier-Cabot, From damage to fracture mechanics and conversely: A combined approach, International Journal of Solids and Structures 33 (2022) (1996) 3327 - 3342, ISSN 0020-7683, doi:10.1016/0020-7683(96)00015-7.

[37] F. Cazes, M. Coret, A. Combescure, A. Gravouil, A thermodynamic method for the construction of a cohesive law from a nonlocal damage model, International Journal of Solids and Structures 46 (6) (2009) 1476 - 1490, ISSN 0020-7683, doi:10.1016/j.ijsolstr.2008.11.019.

[38] C. Comi, S. Mariani, U. Perego, An extended FE strategy for transition from continuum damage to mode I cohesive crack propagation, International Journal for Numerical and Analytical Methods in Geomechanics 31 (2) (2007) 213-238, ISSN 1096-9853, doi:10.1002/nag.537.

[39] S. Cuvilliez, F. Feyel, E. Lorentz, S. Michel-Ponnelle, A finite element approach coupling a continuous gradient damage model and a cohesive zone model within the framework of quasi-brittle failure, Computer Methods in Applied Mechanics and Engineering 237240 (0) (2012) 244 - 259, ISSN 0045-7825, doi:10.1016/j.cma.2012.04.019.

[40] I. Arias, J. Knap, V. B. Chalivendra, S. Hong, M. Ortiz, A. J. Rosakis, Numerical modelling and experimental validation of dynamic fracture events along weak planes, Computer Methods in Applied Mechanics and Engineering 196 (37-40) (2007) 3833 - 3840, ISSN 0045-7825, doi:10.1016/j.cma.2006.10.052.

[41] J. F. Molinari, G. Gazonas, R. Raghupathy, A. Rusinek, F. Zhou, The cohesive element approach to dynamic fragmentation: the question of energy convergence, International Journal for Numerical Methods in Engineering 69 (3) (2007) 484-503, ISSN 1097-0207, doi:10.1002/nme.1777.

[42] J. Mazars, G. Pijaudier-Cabot, Continuum Damage TheoryApplication to Concrete, Journal of Engineering Mechanics 115 (2) (1989) 345 - 365, doi:10.1061/(ASCE)0733-9399(1989)115:2(345).

[43] M. Geers, R. de Borst, W. Brekelmans, R. Peerlings, Validation and internal length scale determination for a gradient damage model: application to short glass-fibre-reinforced polypropylene, International Journal of Solids and Structures 36 (17) (1999) 2557 - 2583, ISSN 0020-7683. 
[44] R. Peerlings, M. Geers, R. de Borst, W. Brekelmans, A critical comparison of nonlocal and gradient-enhanced softening continua, International Journal of Solids and Structures 38 (2001) 7723-7746.

[45] L. Wu, L. Noels, L. Adam, I. Doghri, An implicit-gradient-enhanced incremental-secant mean-field homogenization scheme for elasto-plastic composites with damage, International Journal of Solids and Structures 50 (24) (2013) 3843 - 3860, ISSN 0020-7683.

[46] F. Dufour, G. Pijaudier-Cabot, M. Choinska, A. Huerta, Extraction of a crack opening from a continuous approach using regularized damage models, Computers \& Concrete 5 (4) (2008) 375-388.

[47] A. Pandolfi, M. Ortiz, An Efficient Adaptive Procedure for Three-Dimensional Fragmentation Simulations, Engineering with Computers 18 (2) (2002) 148-159, URL http://dx.doi.org/10.1007/s003660200013.

[48] V. Tvergaard, J. W. Hutchinson, The relation between crack growth resistance and fracture process parameters in elasticplastic solids, Journal of the Mechanics and Physics of Solids 40 (6) (1992) 1377 - 1397, ISSN 0022-5096, doi:10.1016/00225096(92)90020-3.

[49] J. J. Remmers, R. Borst, C. V. Verhoosel, A. Needleman, The cohesive band model: a cohesive surface formulation with stress triaxiality, International Journal of Fracture 181 (2) (2013) 177-188, ISSN 0376-9429, doi:10.1007/s10704-013-98343, URL http://dx.doi.org/10.1007/s10704-013-9834-3.

[50] A. L. Gurson, Continuum Theory of Ductile Rupture by Void Nucleation and Growth: Part I-Yield Criteria and Flow Rules for Porous Ductile Media, Journal of Engineering Materials and Technology 99 (1) (1977) 2-15, doi: $10.1115 / 1.3443401$.

[51] A. McBride, J. Mergheim, A. Javili, P. Steinmann, S. Bargmann, Micro-to-macro transitions for heterogeneous material layers accounting for in-plane stretch, Journal of the Mechanics and Physics of Solids 60 (6) (2012) 1221 - 1239, ISSN 0022-5096, doi:10.1016/j.jmps.2012.01.003.

[52] L. Noels, R. Radovitzky, A general discontinuous Galerkin method for finite hyperelasticity. Formulation and numerical applications, International Journal for Numerical Methods in Engineering 68 (1) (2006) 64-97, ISSN 1097-0207, doi: $10.1002 /$ nme.1699.

[53] A. T. Eyck, A. Lew, Discontinuous Galerkin methods for non-linear elasticity, International Journal for Numerical Methods in Engineering 67 (9) (2006) 1204-1243, ISSN 1097-0207, doi:10.1002/nme.1667.

[54] L. Noels, R. Radovitzky, An explicit discontinuous Galerkin method for non-linear solid dynamics: Formulation, parallel implementation and scalability properties, International Journal for Numerical Methods in Engineering 74 (9) (2008) 1393-1420, ISSN 1097-0207, doi:10.1002/nme.2213.

[55] A. Lew, A. Eyck, R. Rangarajan, Some Applications of Discontinuous Galerkin Methods in Solid Mechanics, IUTAM Symposium on Theoretical, Computational and Modelling Aspects of Inelastic Media (2008) 227-236doi:10.1007/978-14020-9090-5_21.

[56] A. T. Eyck, F. Celiker, A. Lew, Adaptive stabilization of discontinuous Galerkin methods for nonlinear elasticity: Motivation, formulation, and numerical examples, Computer Methods in Applied Mechanics and Engineering 197 (45-48) (2008) 3605-3622, ISSN 0045-7825.

[57] G. M. Hulbert, J. Chung, Explicit time integration algorithms for structural dynamics with optimal numerical dissipation, Computer Methods in Applied Mechanics and Engineering 137 (2) (1996) 175 - 188, ISSN 0045-7825, doi:10.1016/S00457825(96)01036-5.

[58] C. Geuzaine, J.-F. Remacle, Gmsh: A 3-D finite element mesh generator with built-in pre- and post-processing facilities, International Journal for Numerical Methods in Engineering 79 (11) (2009) 1309-1331, doi:10.1002/nme.2579.

[59] L. Noels, R. Radovitzky, A new discontinuous Galerkin method for Kirchhoff-Love shells, Computer Methods in Applied Mechanics and Engineering 197 (33-40) (2008) 2901-2929, ISSN 0045-7825, doi:10.1016/j.cma.2008.01.018.

[60] J.-F. Molinari, Dynamic Fracture: Discrete Versus Continuum Damage Modeling, in: The Third International Conference on Computational Modeling of Fracture and Failure of Materials and Structures, Prague, Czech Republic, 57 June 2013, 2013. 